\title{
Regards croisés sur l'état de la Loire Moyenne : potamoplancton et qualité de l'eau, quel enseignement tirer de 20 années d'études?
}

\author{
Cross overlook on the Middle Loire river status : \\ potamoplankton and water quality, \\ which lessons to draw from twenty years studies?
}

\section{Nicole Lair}

Université Blaise Pascal, UMR CNRS 6042, Equipe Hydrosystèmes \& Bassins Versants, Maison de la Recherche, 63057 Clermont-Ferrand Cedex (France) Nicole.Lair@univ-bp.clermont.fr

Résumé. - La Loire est utilisée par l'homme depuis très longtemps et c'est au début des années soixante qu'Electricité de France s'est préoccupée des nuisances que pouvait apporter la réfrigération de ses centrales thermiques. La mise en place de la Loi relative aux installations classées pour l'environnement, qui date de 1976, a conduit cet organisme à mettre en place des études de surveillance écologique du milieu. Les échantillons recueillis entre juin et octobre, de 1982 à 2001, ont permis de suivre les variations de la qualité de l'eau en relation avec les communautés vivantes. Les résultats obtenus aux trois stations échantillonnées en amont et en aval des sites de Dampierre-en-Burly et de Saint-Laurent-des-Eaux situés de $550 \mathrm{~km}$ à $640 \mathrm{~km}$ des sources du fleuve, ont servi de base de données. Dans le Val de Loire, les écoulements offrent des situations contrastées et les étiages peuvent être très sévères. L'habitat physique est un élément déterminant du développement du potamoplancton ; celui-ci est favorisé par les zones lentiques qui peuvent représenter une large part de la masse d'eau. Les divers apports de nutriments contribuent à un développement massif d'algues planctoniques et, avec des teneurs en chlorophylle $>200 \mu \mathrm{g} \cdot \mathrm{I}^{-1}$, c'est l'un des fleuves européens les plus riches en algues.

L'examen des données recueillies pendant ces vingt années a permis de constater que l'oxygénation de l'eau de ce secteur de fleuve est étroitement liée à la quantité d'algues, les processus de production l'emportent au regard de la demande biochimique en oxygène. Les éléments nutritifs et la silice sont corrélés positivement aux débits. Les nitrates, corrélés négativement à la densité algale, ont été moins consommés au cours des dernières années. Au cours des années hydrologiquement déficitaires, les phosphates ainsi que la silice tendent à devenir limitants. En outre, parallèlement à la baisse des flux de phosphates (en limite de détection depuis 1998), la densité des algues a diminué depuis 1995 (site amont) et depuis 1996 (site aval). Ceci suggère que les efforts d'épuration développés par les Agences de l'Eau portent leurs fruits, mais en conséquence, il s'est produit une diminution concomitante des teneurs en oxygène dissous. 
Ces algues pouvant être régulées par leurs consommateurs potentiels, une étude des rotifères (composants quasi-exclusifs des métazoaires zooplanctoniques vivant en Loire moyenne) s'est ajoutée aux autres variables à partir de 1995. Sur cette période de moyen terme (1995-2001), caractérisée par des eaux relativement basses, les teneurs en oxygène dissous ne sont plus liées aux algues dont la densité est corrélée positivement à la $\mathrm{DBO}_{5}$, ce qui sous entend que les processus de décomposition prennent de l'importance. Durant cette période, tandis qu'une baisse progressive des Chlorophycées se dessine, la corrélation positive entre ces algues vertes et les rotifères, suggère que si ces derniers sont susceptibles de les réguler, la nourriture algale n'est pas limitante. Le potamoplancton demeure dans l'ensemble bien diversifié.

Les rejets d'eau chaude, qui se sont considérablement atténués au cours du temps, de par l'évolution de la conception des nouvelles centrales, ne paraissent pas affecter les communautés vivantes. Au cours de ces dernières années, celles-ci ont été exposées à des perturbations de l'environnement de courte durée (résiduels de chlorations, déplacements de matériaux en suspension). L'environnement local contribue à l'hétérogénéité de distribution du potamoplancton et l'examen attentif de la base de données a permis d'illustrer ses fortes capacités de récupération. L'effet des chlorations sur les algues s'est estompé en moins d'un mois et la diversité spécifique des rotifères s'est rapidement rétablie. Les nuisances liées à différents travaux de terrassement (pont de Loire en amont, pose de diffuseur par EDF, etc.) ont été diversement ressenties : la densité des rotifères a été affectée avant de se rétablir à nouveau et l'impact visuel des films bactériens a perduré localement plusieurs années.

Ces données de base recueillies sur 20 années s'avèrent représenter un outil de travail pertinent pour définir ce que doit être « le bon état écologique » de ce secteur de Loire, à atteindre à l'horizon 2015, pour satisfaire à l'objectif de la Directive Cadre Européenne sur l'eau. Le bilan actuel illustre tout l'intérêt de disposer de longues séries de données : cette étude a montré qu'au cours de ces dernières années il s'est produit une diminution significative de la quantité d'algues, mais aussi des teneurs en oxygène dissous. En outre, elle a permis de mettre en évidence que les communautés potamoplanctoniques demeurent bien diversifiées et que le système biologique, observé dans ce secteur largement eutrophe de Loire Moyenne, est résilient.

Mots clés. - Eaux courantes, Loire, long terme, qualité de l'eau, algues, rotifères.

Abstract. - The river Loire was used by man since a long time and in the sixteen's, Electricité de France was concerned about nuisances that might introduce the cooling of its thermal power plants. The establishment in 1976, of the Law about the listed installations for environment, lead this organism to put ecological surveillance programmes in place. The variations in water quality, extracted from comparable data collected bimonthly from June to October, were followed during 20 years in connection with planktonic communities. The databases are those of three stations sampled up and downstream the power plants of Dampierre-en-Burly and Saint-Laurent-des-Eaux, situated at $550 \mathrm{~km}$ and $640 \mathrm{~km}$ from the springs. The "Val de Loire" is characterised by sharply contrasting flows, with severe low waters. The physical habitat is a determining factor in the potamoplankton growth, which is favoured by lentic areas that can represent large surfaces. With chlorophyll a measures that can reach values higher than $200 \mu \mathrm{g} . \mathrm{I}^{-1}$, the Middle Loire was found among the richer European rivers.

The examination of the data collected from 1982 to 2001 established that water oxygenation was closely linked to the algal density and, related to the $\mathrm{BOD}_{5}$, the production processes were prevailing. Nutrient and silicate were positively correlated to the river 
discharge. The nitrates were negatively correlated to the algal density, but less consumed for the last years. The phosphates that were beyond the limits of detection since 1998, tended to be limiting and the silica too. The fluxes in phosphates and algae have decreased parallel to the algal density and the dissolved oxygen, which suggests that catchment area treatments performed by the Loire-Bretagne Water Agency authorities were fruitful.

Moreover, a study of the rotifers was added since 1995 due to their potential control on algae, This medium-term period (1995-2001) was characterised by low-waters, the dissolved oxygen were no more correlated to algae whose density was positively correlated to $\mathrm{BOD}_{5}$, showing that decomposition processes gained in importance, contrary to the primary production evolution over the oxygen balance. During this period, while the Chlorophytes tended to decrease, their positive correlation with rotifers suggested that these filter-feeders, which were not food limited, could regulate the algae. The potamoplankton remained very well diversified.

The heated water discharge, considerably attenuated with time due to improvement in cooling systems of power stations, did not seem affect the living communities that have been exposed to short-term disturbances (chlorination residues, increase in suspended matter). The local environment was in part responsible for the distribution heterogeneity of the living material and the detailed examination of the database allowed illustrating their high recovery capacity. Algae recuperated from the chlorination disturbance in less that one-month and the specific rotifers diversity was quickly re-established. The disturbances due to the earthworks in the riverbed were variously received, the rotifers density was affected before to be re-established and bacterial films were locally visible for several years.

The 20 years data are a pertinent tool to specify what is "the good ecological condition" of the Middle Loire, which had to be reached on the 2015 horizon in the frame of the Water European Directive. The actual assessment allows saying that the biological system in this largely eutrophic section of the Middle Loire was resilient and the community well diversified. In contrast, the decrease in algae that came last leaded to a decrease in dissolved oxygen, such results showing the public interest to have long term series data.

Key words. - Running waters, Loire, long term, water quality, algae, rotifers.

\section{INTRODUCTION}

\subsection{Loire d'hier et d'aujourd'hui}

Evoquer le livre consacré aux "Voyages sur la Loire. A plaisir et à gré le vent... »(Comte, 1998), c'est se souvenir que l'homme utilise les eaux de ce fleuve depuis bien longtemps et « Le fleuve lui-même a perdu son caractère sauvage depuis au moins huit siècles" (Schulé, 2000). Mais depuis les temps historiques de la navigation ligérienne, qui cessa au milieu du XIX siècle, la révolution industrielle, la révolution verte, l'augmentation démographique et de la demande énergétique ont accru les besoins en eau et contribué à une utilisation croissante de cette ressource renouvelable, mais limitée. Pourtant, la prise de conscience des excès auxquels les eaux douces intérieures ont été soumises est récente; ainsi à la fin des années soixante, la perception d'un fleuve au service de l'homme était toujours d'actualité : 
"Malgré sa longueur de 1020 km, malgré les ramifications de ses affluents qui drainent un bassin de $115000 \mathrm{~km}^{2}$ situé au centre du pays, et malgré un débit moyen de $840 \mathrm{~m}^{3} / \mathrm{s}$ qui la classe au second rang après le Rhône, la Loire est de tous les fleuves français le plus mal utilisé à l'heure actuelle... » (Fénelon, 1967). La construction de barrages-réservoirs coulait sous le sens: "Tout d'abord il est indispensable d'améliorer le débit du fleuve et de ses affluents en atténuant aussi bien les étiages que les crues. Pour obtenir ce double résultat, seuls les barragesréservoirs peuvent jouer un rôle efficace". II était aussi question de "l'ordonnance du 7.1.59, renouvelant le décret du 11.9.31 reconnaissant d'utilité publique le captage des eaux du Val de Loire par Paris ». Ou encore, à propos de l'irrigation " 60 , 100 ou 140 mètres cubes par seconde sous les ponts de Gien, c'est encore une Loire bienfaisante et non plus un fleuve inutilisable et dangereux qui traversera vers 1975 un Val d'Orléans prospère ". Ou, concernant les aménagements touristiques, «Pour atténuer les inconvénients que présente pour le visiteur une Loire non aménagée, les divers organismes qui s'intéressent au fleuve, et tout particulièrement les municipalités des grandes villes, cherchent à discipliner les eaux qui passent près de leurs agglomérations et à parfaire leur agrément ». Enfin, au sujet des pollutions et de la consommation, "Le Comité de Loire s'en est inquiété... On a constaté avec satisfaction qu'en une dizaine de kilomètres se produit un phénomène d'autoépuration. Mais ce n'est pas suffisant si l'on veut utiliser également la Loire et ses affluents pour l'adduction d'eau des villes et pour les agréments touristiques. Des stations d'épuration sont prévues à... » (Fénelon, 1967). Ainsi, suffisait-il à l'époque, de "domestiquer » le fleuve et ses affluents (Gachon, 1964), ce à quoi s'employèrent activement Services Publics et Privés, Comités et Associations, aidés par les Universités. Sans oublier le manque de connaissances en matière de dynamique et d'écologie fluviale; était-ce parce que la nature n'avait pas atteint ce seuil limite qui rend les choses suffisamment visibles pour que chacun s'en inquiète? Le Plan Loire Grandeur Nature ne verra le jour qu'à la fin des années quatre-vingt (Schulé, 2000).

\subsection{Les lois sur l'eau}

En 1971, préoccupée par l'eutrophisation, la Direction de l'Environ(nement de l'Organisation de Coopération et de Développement Economiques (O.C.D.E.) lançait un programme de coopération pour la surveillance des eaux continentales lacustres, à partir d'un protocole de mesures commun aux laboratoires participants appartenant à divers pays, dont la France (Lair, 19751976 ; O.E.C.D., 1982). En France, un Ministère de l'Environnement était créé au début des années soixantedix, siégeant à ses débuts au Minis- 
tère de la Marine. II va sans dire que les études, les recherches et la mise au point des méthodes de contrôle se multipliaient. La loi de protection de la nature du 10 juillet 1976, suivie le 19 juillet de la loi relative aux installations classées pour l'environnement, va faire suite à la loi du 16 décembre 1964, relative au régime et à la répartition des eaux. Sous le contrôle des Agences Financières de Bassin instituées en 1964 (et devenues depuis Agences de l'Eau), diverses actions ont eu pour but de faciliter l'installation de stations d'épuration, de manière à limiter les rejets des éléments nutritifs responsables des processus d'eutrophisation. La loi sur l'eau du 3 janvier 1992 insistait - entre autres aspects - sur les actions à mener pour restaurer la qualité des eaux superficielles, anticipant ainsi la future Directive Cadre Européenne (DCE) de 1998.

Par ailleurs, dès le début des années soixante, Electricité de France s'est préoccupée des nuisances que pouvait apporter la réfrigération des centrales thermiques en circuit ouvert implantées sur les grands cours d'eau français, dont la centrale de Montereau construite en 1960-1962 (EDF, 1996). Le Comité Scientifique de Montereau, nouvellement créé, s'était chargé de programmer les études qui se sont déroulées de 1962 à 1978 (Khalanski, Leynaud \& Perez, rapport EDF-DER, 1978). II faut dire qu'à l'époque, les réchauffements provoqués par les installations étaient importants, comparativement aux rejets actuels.
En 1967, la Mission Technique de l'Eau Loire-Bretagne s'intéressait aux modifications que pourrait apporter au milieu la mise en service de la centrale nucléaire de Saint-Laurent-desEaux. Aguesse, chargé de dresser l'état biologique des eaux, faisait état de l'abondance des matières organiques au regard des espèces présentes et des valeurs de $\mathrm{DBO}_{5}$ et de $\mathrm{DCO}$ (Aguesse, 1968, rapport du Laboratoire d'Ecologie de la faculté des Sciences d'Orléans). En 1969, le Conseil Supérieur de la Pêche se préoccupait des proliférations de rotifères par rapport au réchauffement des eaux de la Loire (Chancerel, 1972, rapport du CSP de Poitiers) et le département Environnement Aquatique et Atmosphérique dépendant de la Direction des Etudes et Recherches EDF, à l'appui du Comité Scientifique de Montereau, continuait d'encourager les études sur ce sujet (Pourriot et al., 1972 ; Champ, 1977 ; 1978). En été 1976 , la sécheresse exceptionnelle conduisait EDF à initier une étude hydrobiologique sur l'un de ses sites électronucléaires ligériens (Saint-Laurent-des-Eaux) (Lair et al., 1978).

Dès le début de l'année 1977, en vue de l'application de la loi sur les études d'impact, EDF engageait des études de «point zéro » pour préparer les demandes d'autorisation relatives à la construction de nouvelles centrales électronucléaires. Les sites implantés en Loire, représentant $20 \%$ de l'ensemble du parc nucléaire français, couvrent un tronçon de plus de $250 \mathrm{~km}$, allant de Belleville-sur- 
Loire à Chinon (Lair et al., 1980). L'état des lieux en Loire Moyenne fut dressé en 1977 et 1978, puis les études de surveillance écologique se sont enchaînées au fil des années, prenant en compte l'évolution des techniques d'analyse et des connaissances (Lair \& Sargos, 1993 ; Lair et al., 1996 ; Lair \& Reyes-Marchant, 2000).

\subsection{Les données disponibles}

Nous disposons à ce jour d'une banque de données comparables sur 20 ans, permettant d'observer les changements survenus dans la qualité de l'eau de ce secteur de Loire, elle-même liée aux communautés vivantes. II sera question de deux sites (Dampierre-en-Burly et Saint-Laurentdes-Eaux), pour lesquels le jeu de données recueillies depuis 1982 est comparable. Dans le présent article, ce sont les résultats observés sur l'ensemble des communautés planctoniques, en relation avec les variables de l'environnement abiotique liées à leur métabolisme global, qui seront privilégiés et non le devenir des espèces.

Les organismes vivants sont fréquemment exposés à des perturbations de l'environnement (naturelles et anthropiques), de courte durée (traitements et travaux divers), ou de longue durée (eutrophisation) et certains d'entre eux peuvent être de bons indicateurs des "restaurations écologiques ». C'est en ce sens que nous porterons un regard global sur cette période de 20 années, de ma- nière à suivre l'évolution temporelle de la qualité des eaux de ce secteur de fleuve. Les processus biologiques étant liés aux ressources nutritives, limitantes ou non, ainsi qu'aux effets de la prédation invertébrée et/ou vertébrée, ils peuvent entraîner des changements spatiaux et temporels considérables (Bunn \& Davies, 2000). Aussi nous examinerons, sur le moyen-terme, le fonctionnement écotrophique des communautés planctoniques (incluant les rotifères étudiés depuis 1995), à partir des données bimensuelles recueillies entre juin et octobre de 1995 à 2001. Enfin, nous changerons d'échelle pour porter un regard attentif sur les données détaillées recueillies sur chaque site, afin de discuter de l'impact que diverses nuisances (thermiques, chlorations résiduelles, travaux de terrassement effectués en amont ou sur les sites) sont susceptibles de provoquer.

\section{MÉTHODES ET DESCRIPTION DES SITES}

\subsection{Caractéristiques générales de la Loire moyenne}

Le fleuve lui-même offre des situations très contrastées. Les écoulements de Loire sont aussi variables qu'imprévisibles et si les débits d'étiage sont actuellement soutenus à $60 \mathrm{~m}^{3} \cdot \mathrm{s}^{-1}$ par les barrages EDF situés en amont (Lair et al. 1996), le record de crues avait atteint $7600 \mathrm{~m}^{3} \cdot \mathrm{s}^{-1}$ au Bec d'Allier en 1856 et seulement $19 \mathrm{~m}^{3} \cdot \mathrm{s}^{-1}$ avaient été mesurés à Blois 


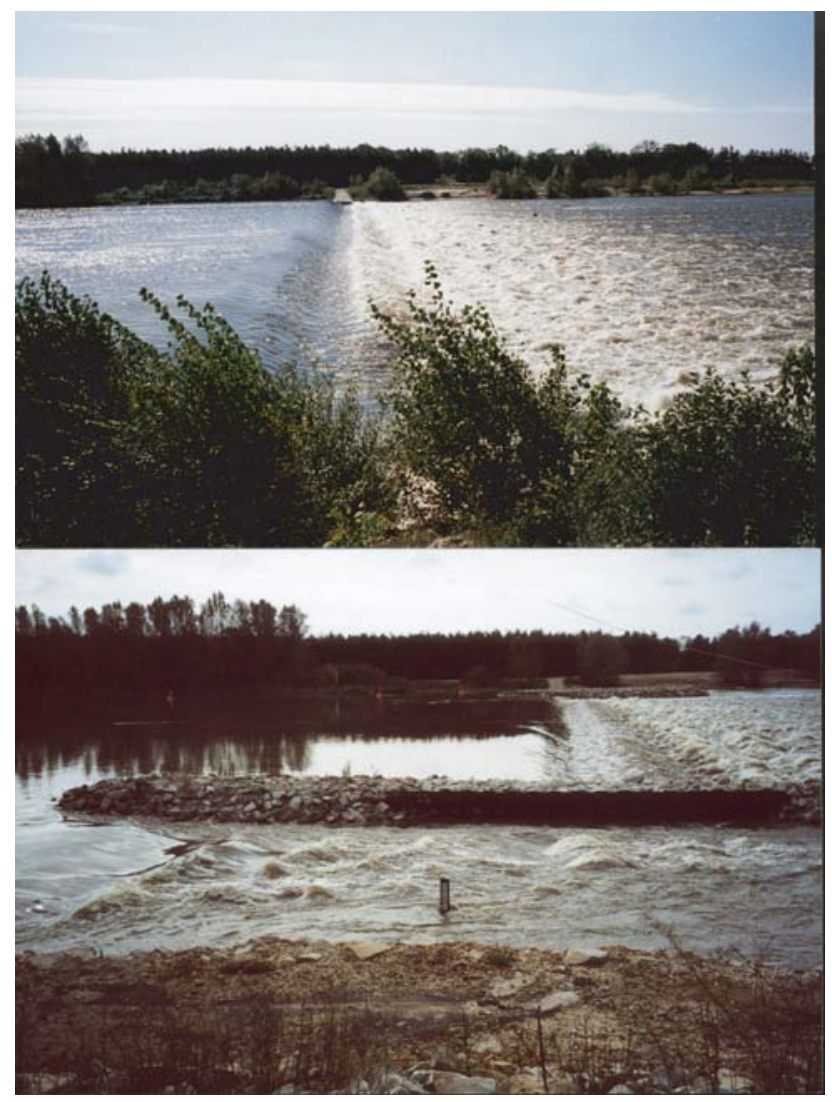

Photo 1. - La Loire au niveau du seuil de Dampierre-en-Burly en juin 1995 (vue supérieure) et en octobre 2000 (vue inférieure) en période de hautes eaux. Clichés P. Reyes-Marchant.

Photo 1. - The middle Loire at the level of the bank of Dampierre-en-Burly during a high-water period (upper slide, June 1995, lower slide October 2000). Snapshots from P. Reyes-Marchant

en 1949 (Schulé, 2000). Ces classiques variations de débit sont la cause de l'hétérogénéité intra- et inter- saisonnière (Lair \& Sargos, 1993) et, quand le courant est fort (Photo 1), mesurer des différences entre une station de référence, située en amont des installations, et des stations situées en aval, relève de la gageure. Au regard de l'hétérogénéité inter-annuelle du régime hydraulique de ce secteur de fleuve, ceci a conduit à rechercher une période moins sujette à des crues de grande ampleur, de manière à prendre en compte les processus locaux inhérents à la géomorphologie propre à la Loire Moyenne. En effet, la structure des communautés d'eaux courantes est déterminée par la nature de l'habitat physique, un habitat par essence hétérogène et souvent négligé en tant que facteur 
de forçage (Bunn \& Davies, 2000), d'autant que les communautés d'eaux courantes ont de remarquables capacités de dispersion.

\subsection{Les sites d'étude}

Les méthodes utilisées ont été largement décrites dans les documents de synthèse consacrés à Dampierreen-Burly, qui se trouve dans le Val de Loire à $550 \mathrm{~km}$ de la source du fleuve et à Saint-Laurent-des-Eaux, situé $90 \mathrm{~km}$ en aval, (Lair et al., 1996 ; Lair \& Reyes-Marchant, 2000), (Fig. 1). Le secteur étudié est soumis à la confluence Loire- Allier et le débit annuel décennal s'étend entre 200 et $460 \mathrm{~m}^{3} \cdot \mathrm{s}^{-1}$ à Gien (situé en amont de Dampierre-en-Burly) et entre 235 et 485 à Blois (situé en aval de SaintLaurent-des-Eaux), (Schulé, 2000).
En l'absence d'affluent notable entre les deux sites, les débits mesurés à Dampierre-en-Burly et à Saint-Laurent-des-Eaux varient en parallèle $(r=0,90)$ et le module naturel augmente très peu en aval. Dans ce secteur de «la boucle orléanaise », une partie des débits circule souterrainement et la nappe des calcaires de Beauce contribue à compenser les pertes d'eau situées dans le lit (Schulé, 2000).

Sur chaque site, trois stations sont échantillonnées, l'une en amont des installations, la seconde en aval immédiat des rejets et la troisième en aval plus éloigné, là où les rejets sont pleinement dilués (Fig. 2). Outre les mesures in situ, les analyses physico-chimiques ont été régulièrement effectuées selon les normes en vigueur, dans des laboratoires d'analyse agréés.

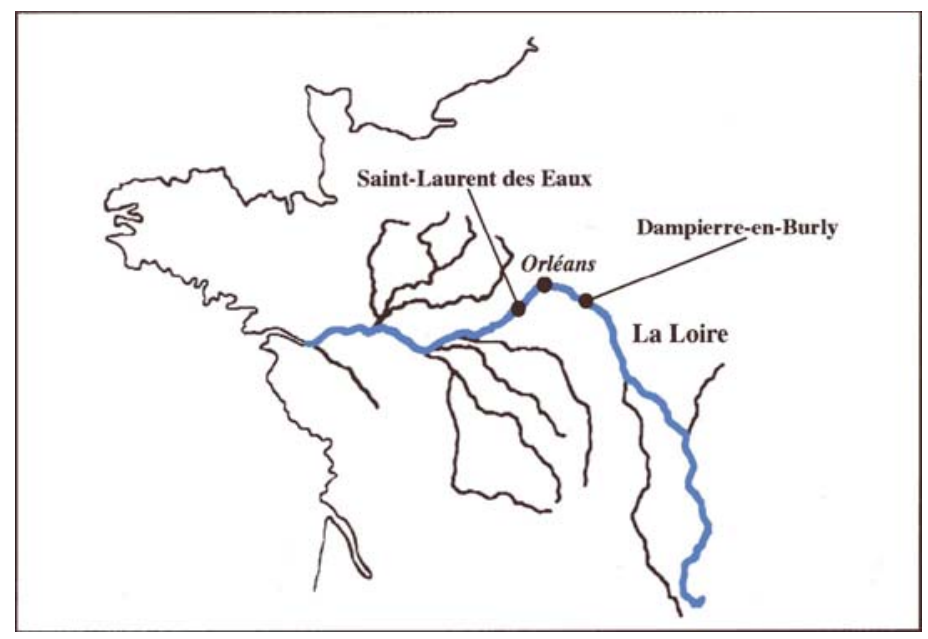

Fig. 1. - Position des sites de Loire présentés dans ce travail.

Fig. 1. - Location of the two sites in the course of the River Loire. 


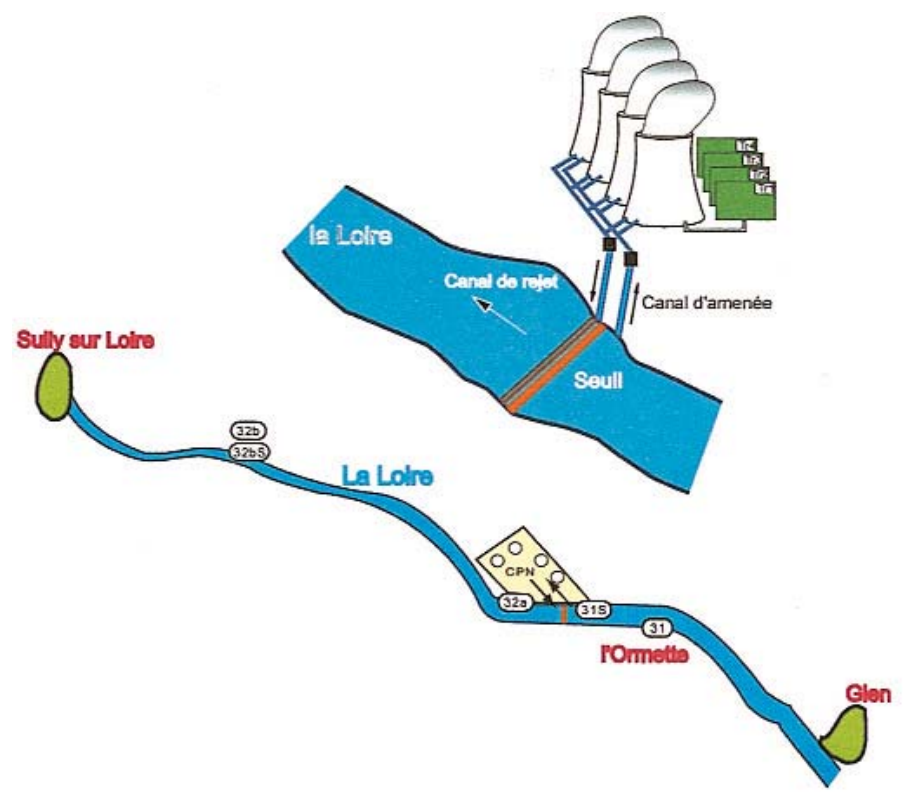

Fig. 2. - Position des trois stations d'étude sur le site de la centrale de Dampierre-en-Burly $(31=$ station amont, $32 \mathrm{a}=$ station à l'aval immédiat du rejet, $32 \mathrm{~b}=$ station plus éloignée en aval).

Fig. 2. - Location of the three sampling stations on the site of Dampierre-en-Burly (31 is upstream, $32 \mathrm{a}$ is just after the power plant, $32 \mathrm{~b}$ is more downstream).

L'expérience acquise au cours de la réalisation des programmes de surveillance nous a conduit à privilégier la période présumée d'étiage, qui est une période d'autant plus sensible qu'elle est associée à des températures élevées. Les variations mensuelles des débits mesurés au droit de Saint-Laurent-des-Eaux et calculés à partir des relevés journaliers sur la période 1976-2001 illustrent la fréquence des hautes eaux en mai. (Fig. 3). Ainsi, dans un souci d'homogénéisation, seules les données recueillies entre juin et octobre (ce qui est le cas pour l'ensemble du « moyen terme ») ont été analysées dans cette étude, l'expression « année », dé- signant en fait cette période, dite sensible.

En Loire moyenne, les étiages, même soutenus, peuvent être très sévères et, en plein été, les processus hydrauliques inhérents à la fois à la baisse du niveau de l'eau (Photo 2), à la vitesse variable du courant et à l'action du vent, induisent inévitablement un mélange des eaux de surface, dont l'importance est grande au regard du développement des communautés vivantes (Weithoff et al., 2000).

De tels phénomènes s'observent facilement à l'œil nu. A certaines périodes, le sens du courant est difficile- 

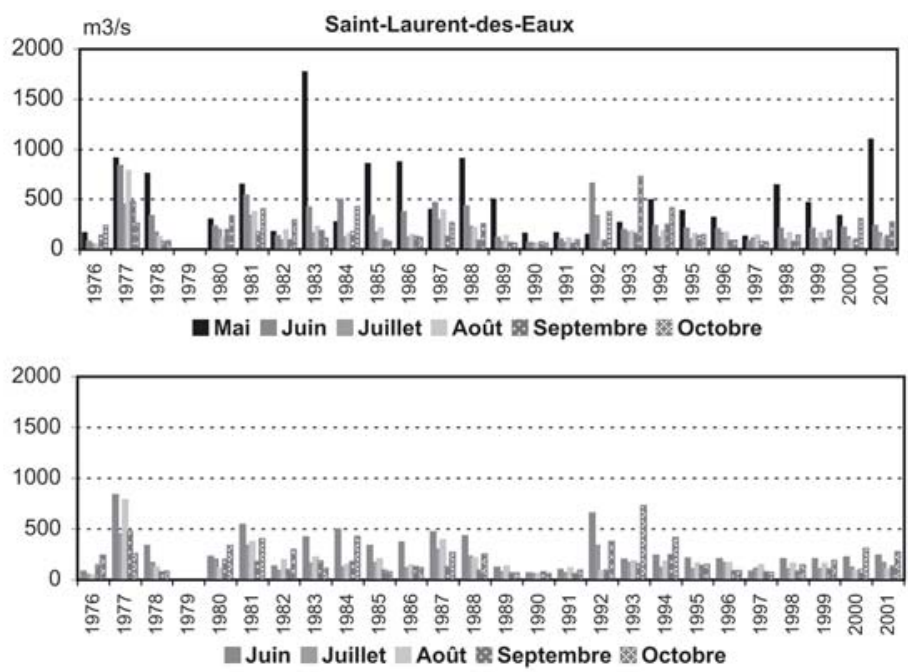

Fig. 3. - Comparaison des variations de débits $\left(\mathrm{m}^{3} . \mathrm{s}^{-1}\right)$ relevés dans le secteur de Saint-Laurentdes-Eaux de 1976 à 2001. Les moyennes mensuelles ont été calculées à partir des débits journaliers.

Fig. 3. - Comparison of the variations in water discharge $\left(\mathrm{m}^{3} \cdot \mathrm{s}^{-1}\right)$. Monthly data were calculated from daily measurements.

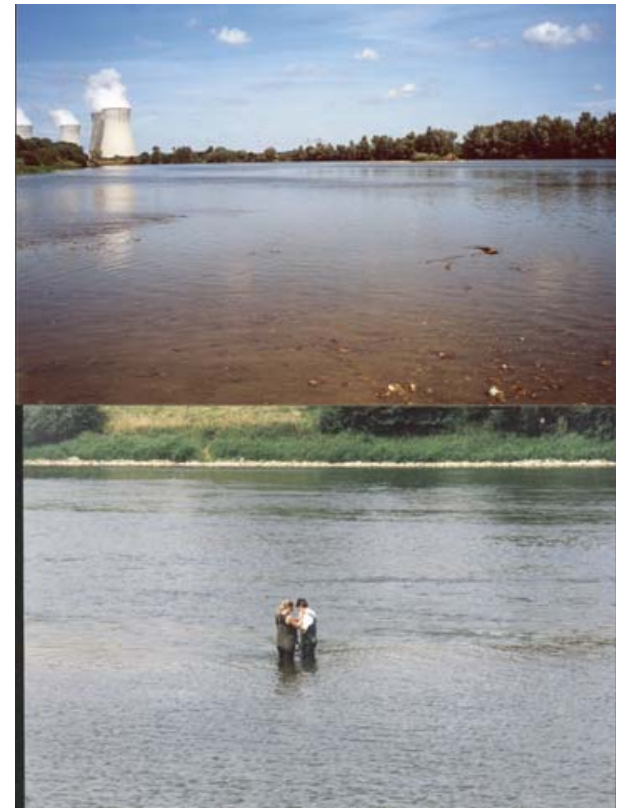

Photo 2. - Etiages en Loire Moyenne, les processus locaux l'emportent sur les processus de transfert amont-aval. Clichés P. Reyes-Marchant.

Photo 2. - Low waters in the Middle Loire, local processes are important in comparison with updownstream transfers. Snapshots from P. Reyes-Marchant. 
ment perceptible en dehors du chenal, dont la taille peut être réduite à quelques mètres de largeur. C'est ainsi que les communautés vivantes, dont le développement est favorisé dans les zones lentiques, se mêlent localement aux communautés en dérive, accentuant l'effet de l'hétérogénéité. De plus, les turbulences d'amplitude modérée favorisent la croissance algale (Köhler, 1997). De ce fait, en plein étiage, dans les zones de méandres typiques de ce Val de Loire, une large part de la masse d'eau est " en quelque sorte immobile » et, pratiquement, l'ensemble

éclairé jusqu'au fond, devient très productif. C'est aussi parce que le secteur étudié se trouve à plus de $500 \mathrm{~km}$ de la source, parce que les apports nutritifs liés aux activités humaines s'ajoutent aux apports naturels qui dérivent depuis l'amont, que les algues planctoniques y sont très denses (Photo 3).

Tout cela explique pourquoi la Loire est l'un des fleuves européens, qui contient les plus fortes teneurs en chlorophylle $a$, dont les valeurs peuvent dépasser ponctuellement les $200 \mu \mathrm{g} \cdot \mathrm{l}^{-1}$ (un maximum de $260 \mu \mathrm{g} \cdot \mathrm{I}^{-1}$ a été mesuré en août 1999 en aval de

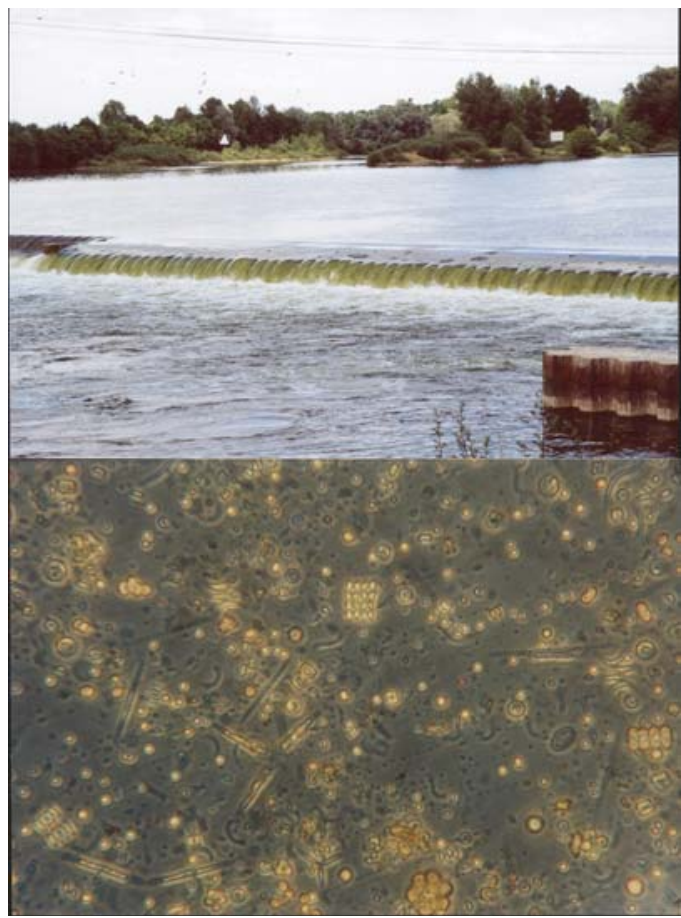

Photo 3. - La Loire Moyenne est un fleuve eutrophe. L'observation microscopique révèle la présence de nombreuses Chlorophytes, accompagnées de Bacillariophycées. Clichés P. Reyes-Marchant.

Photo 3. - The Middle Loire is an eutrophic river. The microscopic observation showed numerous Chlorophytes and Bacillariophytes in addition. Snapshots from P. Reyes-Marchant. 
Tableau I. - Caractéristiques générales du secteur étudié. * (Lair \& Reyes-Marchant, 1997), ** (Lair et al., 1999), ${ }^{* * \star}$ (Lair et al., 1996 ; Lair \& Reyes-Marchant, 1997).

Table I. - General characteristics of the studied area. * (Lair \& Reyes-Marchant, 1997), ** (Lair et al., 1999), ${ }^{* * *}$ (Lair et al., 1996 ; Lair \& Reyes-Marchant, 1997).

\begin{tabular}{|c|c|c|c|c|c|c|}
\hline \multirow[b]{2}{*}{$\begin{array}{l}\text { Distance depuis la source }(\mathrm{km}) \\
\text { Altitude }(\mathrm{m}) \\
\text { Largeur moyenne }(\mathrm{m})\end{array}$} & \multicolumn{3}{|c|}{ Dampierre-en-Burly } & \multicolumn{3}{|c|}{ Saint-Laurent des Eaux } \\
\hline & & $\begin{array}{l}550 \\
123 \\
300\end{array}$ & & \multicolumn{3}{|c|}{$\begin{array}{c}85 \\
200-325\end{array}$} \\
\hline Périodes juin - octobre (1982-2001) & minimum & moyenne & maximum & minimum & moyenne & maximum \\
\hline Débits moyens mesurés entre juin et octobre $(\mathrm{m} 3 / \mathrm{s})$ & 48 & 155 & 771 & 47 & 163 & 864 \\
\hline Température $\left({ }^{\circ} \mathrm{C}\right)$ & 9,5 & 20 & 29,2 & 9,6 & 20.8 & 31,1 \\
\hline $\mathrm{pH}$ & 7,6 & 8,9 & 9,7 & 7,6 & 8,8 & 9,7 \\
\hline $\mathrm{O} 2(\mathrm{mg} / \mathrm{l})$ & 6 & 11 & 19 & 6 & 11 & 18 \\
\hline DBO5 (mg/l) & 1,3 & 4,7 & 9,7 & 0,6 & 5,6 & 12 \\
\hline $\mathrm{NO} 3(\mathrm{mg} / \mathrm{l})$ & $<1,0$ & 4 & 11,8 & $<1,0$ & 3,6 & 11,1 \\
\hline PO4 $(\mu \mathrm{g} / \mathrm{l})$ & $<0,02$ & 0,13 & 0,48 & $<0,02$ & 0,12 & 0.6 \\
\hline $\mathrm{SiO} 2(\mathrm{mg} / \mathrm{l})$ & 0,5 & 6.4 & 14,1 & 0.4 & 6.4 & 14,1 \\
\hline Densité algale $\left(10^{6}\right.$ cells $\left./ \mathrm{l}\right)$ & 1.3 & 50,7 & 189 & 1.6 & 57,4 & 194 \\
\hline Biomasse algale $(\mathrm{mg} / \mathrm{l})$ & 0.50 & 12,91 & 40,80 & 0,46 & 14,95 & 60,82 \\
\hline \multicolumn{7}{|l|}{ Périodes juin - octobre (1995-2001) } \\
\hline Profondeur de disparition du disque de Secchi $(\mathrm{cm})$ & 40 & 59 & 101 & 30 & 56 & 105 \\
\hline Teneurs en chlorophylle a $(\mu \mathrm{g} / \mathrm{l})(1995-2001)$ & 30 & 111 & 238 & 30 & 92 & 197 \\
\hline Densité en rotifères (1995-2000) & 250 & 1018 & 3400 & 167 & 1055 & 4813 \\
\hline \multicolumn{7}{|l|}{ Périodes juin - octobre (données ponctuelles) } \\
\hline Densité des bactéries $10^{\circ}$ cells/l $\left(1999^{* *}\right)$ & 6 & 9 & 11 & 6 & 10 & 13 \\
\hline Densité des flagellés $10^{3}$ cells $/ 1\left(1997^{*} \& 1999^{* *}\right)$ & & $260-4000$ & $700-6800$ & & $880-4400$ & $2400-7500$ \\
\hline Densité des ciliés $10^{3}$ cells $/ l\left(1996-1997^{* \cdots *} \& 1999^{* *}\right)$ & & $12-45$ & $25-85$ & & $23-51$ & $49-99$ \\
\hline
\end{tabular}

Saint-Laurent-des-Eaux). Les caractéristiques générales des deux sites (Tab. I) ont été reprises des documents de synthèse (Lair et al. 1996 ; Lair \& Reyes-Marchant, 2000) et actualisées avec les données recueillies jusqu'en 2001.

\section{RÉSULTATS ET DISCUSSION}

\subsection{Premier regard : le long terme (1982-2001)}

\subsubsection{Variables physico-chimiques et potamoplancton}

Caractéristiques générales

L'état de référence a été difficile à établir en raison d'années hydrologiques atypiques par rapport à la nor- male. En 1976, par suite d'une sécheresse généralisée à la France, l'étiage de la Loire fut particulièrement sévère. L'année suivante, alors que les études nécessaires à l'établissement d'un état de référence débutaient, il n'y a pas eu d'étiage ; les hautes eaux ont persisté jusqu'en juin 1978 et une fois installées, les basses eaux ont duré jusqu'en décembre. Or, les phénomènes biologiques en dépendent, comme le montre la distribution contrastée entre débits et densité algale, avec des corrélations négatives, hautement significatives, y compris avec la biomasse algale (Fig. 4 ; Tab. II).

Classiquement, l'oxygénation de l'eau est la résultante de l'action des brassages, de la photosynthèse, de la respiration et des processus de 

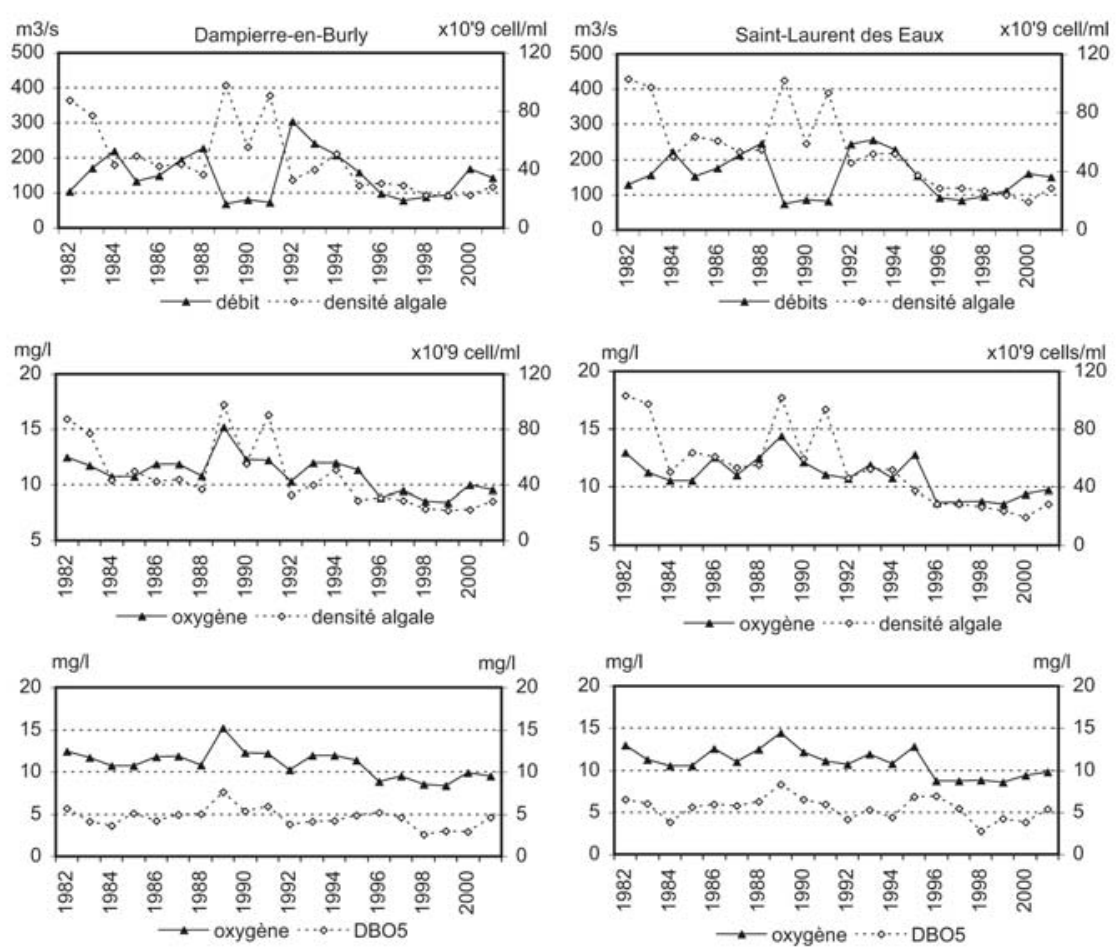

Fig. 4. - Variations des débits, de la densité algale $\left(\times 10^{9} \mathrm{cell}^{\mathrm{ml}}{ }^{-1}\right)$ et des teneurs en oxygène dissous, pour la période 1982-2001.

Fig. 4. - Variations in the water discharge, algal density $\left(\times 10^{9} \mathrm{cell}^{\left.-\mathrm{ml}^{-1}\right)}\right.$ and dissolved oxygen, for the period 1982-2001.

dégradation de la matière organique et, dans de nombreuses rivières à faible pente, durant la période de production, les algues représentent la source principale d'oxygène (Reynolds \& Descy, 1996 ; Wehr \& Descy, 1998). Il est certain que les processus sont complexes, d'autant que la production primaire, classiquement mesurée dans des conditions confinées, donne une image éloignée de la réalité, les algues transportées dans le courant étant soumises à des condi- tions d'éclairement très variables. En Loire moyenne, sur l'ensemble de la période 1982-2001, il n'y a pas de corrélation entre les débits et la quantité d'oxygène dissous; par contre, l'oxygénation de l'eau est étroitement liée à la quantité d'algues, ce que confirment les corrélations positives et hautement significatives entre ces deux variables, illustrant l'importance de la photosynthèse dans le maintien de l'oxygénation. La demande biochimique en oxygène est corrélée positi- 
Tableau II. - Corrélations entre variables, calculées à partir des données recueillies aux trois stations de chaque site durant la période 1982-2001.

Table II. - Correlation between variables, extracted from data collected at each station and site, for the period 1982-2001.

\begin{tabular}{|c|c|c|c|c|c|c|}
\hline \multicolumn{2}{|l|}{ Variables corrélées } & \multirow{2}{*}{$\frac{\text { Dam }}{-0,41}$} & \multirow{2}{*}{$\frac{\text { Slb }}{-0,35}$} & \multirow[b]{2}{*}{ débits /PO4 } & \multirow{2}{*}{$\begin{array}{c}\text { Dam } \\
0,36\end{array}$} & \multirow{2}{*}{$\begin{array}{l}\text { SIb } \\
0,43\end{array}$} \\
\hline$(1982-2001)$ & débits $/ \mathrm{N}$ algues & & & & & \\
\hline étiages sévères & & $-0,33$ & $\#$ & & $\#$ & \# \\
\hline autres étiages & & $-0,53$ & $-0,49$ & & 0,44 & 0,56 \\
\hline (1982-2001) & débits / $\mathrm{B}$ algues & $-0,48$ & $-0,39$ & NO3/N algues & $-0,68$ & $-0,59$ \\
\hline étiages sévères & & $-0,44$ & $\#$ & & $-0,58$ & $-0,44$ \\
\hline autres étiages & & $-0,62$ & $-0,52$ & & $-0,81$ & $-0,40$ \\
\hline (1982-2001) & O2/débits & \# & \# & $\mathrm{PO} 4 / \mathrm{N}$ algues & $-0,30$ & $-0,32$ \\
\hline étiages sévères & & \# & \# & & $\#$ & $\#$ \\
\hline autres étiages & & -035 & $-0,31$ & & $-0,50$ & $-0,43$ \\
\hline$(1982-2001)$ & $\mathrm{O} 2 / \mathrm{N}$ algues & 0,61 & 0,59 & débits / $/ \mathrm{SiO} 2$ & 0,64 & 0,54 \\
\hline étiages sévères & & 0,65 & 0,58 & & $\#$ & $\#$ \\
\hline autres étiages & & 0,57 & 0,63 & & 0,68 & 0,66 \\
\hline$(1982-2001)$ & $\mathrm{O} 2 / \mathrm{B}$ algues & 0,48 & 0,51 & $\mathrm{SiO} 2 / \mathrm{N}$ diatomées & $-0,42$ & $-0,44$ \\
\hline étiages sévères & & 0,54 & 0,49 & & $.0,30$ & $\#$ \\
\hline autres étiages & & 0,42 & 0,55 & & $-0,57$ & $-0,61$ \\
\hline (1982-2001) & $\mathrm{DBO} 5 / \mathrm{O} 2$ & 0,54 & 0,52 & DBO5/N algues & 0,72 & 0,60 \\
\hline étiages sévères & & 0,62 & 0,53 & & 0,69 & 0,49 \\
\hline autres étiages & & 0,49 & 0,56 & & 0,75 & 0,71 \\
\hline (1982-2001) & $\mathrm{O} 2 / \mathrm{NH}_{4}$ & $-0,43$ & $-0,44$ & DBO5/B algues & 0,66 & 0,55 \\
\hline étiages sévères & & $-0,60$ & $-0,89$ & & 0,65 & 0,39 \\
\hline autres étiages & & \# & \# & & 0,68 & 0,69 \\
\hline (1982-2001) & O2/NO3 & $-0,36$ & $-0,23$ & $\mathrm{DBO} 5 / \mathrm{NO} 3$ & $-0,64$ & $-0,57$ \\
\hline étiages sévères & & $\#$ & \# & & $-0,55$ & $-0,31$ \\
\hline autres étiages & & $-0,55$ & $-0,41$ & & $-0,68$ & $-0,68$ \\
\hline (1982-2001) & débits $/ N^{\prime} 3$ & 0,58 & 0,64 & DBO5/NH4 & $\#$ & $\#$ \\
\hline étiages sévères & & 0,65 & 0,57 & & $-0,40$ & $-0,38$ \\
\hline autres étiages & & 0,62 & 0,61 & & $\#$ & $-0,33$ \\
\hline
\end{tabular}

vement aux teneurs en $\mathrm{O}_{2}$ dissous, indiquant que les processus de consommation sont étroitement liés aux processus de production, la production bactérienne étant classiquement supportée par l'excrétion algale et la lyse cellulaire (Servais, 1989). Les corrélations négatives entre $\mathrm{O}_{2}$ et $\mathrm{NH} 4$, entre $\mathrm{O}_{2}$ et $\mathrm{NO} 3$ suggèrent que les processus de nitrification des composés azotés sont rapides. (Fig. 4 ; Tab. II).

Les corrélations positives entre les débits et les éléments nutritifs sont hautement significatives (et accentuées en aval). Les nitrates, qui sont corrélés négativement à la densité (et à la biomasse) algale, ont été large- ment consommés en 1982 et en 1989-91; ils l'ont été notablement moins au cours de ces dernières années, à l'exception de 1997. Ceci se confirme sur les deux sites (Fig. 5). Les phosphates, qui sont également corrélés négativement avec la densité (et la biomasse) algale, paraissent amorcer une nette diminution depuis 1998, les teneurs mesurées à certaines dates étant à la limite de détection de la méthode normalisée d'analyse $\left(<0,02 \mathrm{mg} \cdot \mathrm{I}^{-1}\right)$. Les variations des teneurs en $\mathrm{SiO}_{2}$ sont aussi corrélées positivement aux variations de débits. La densité des diatomées est corrélée négativement à cet élément, essentiel pour la construction 

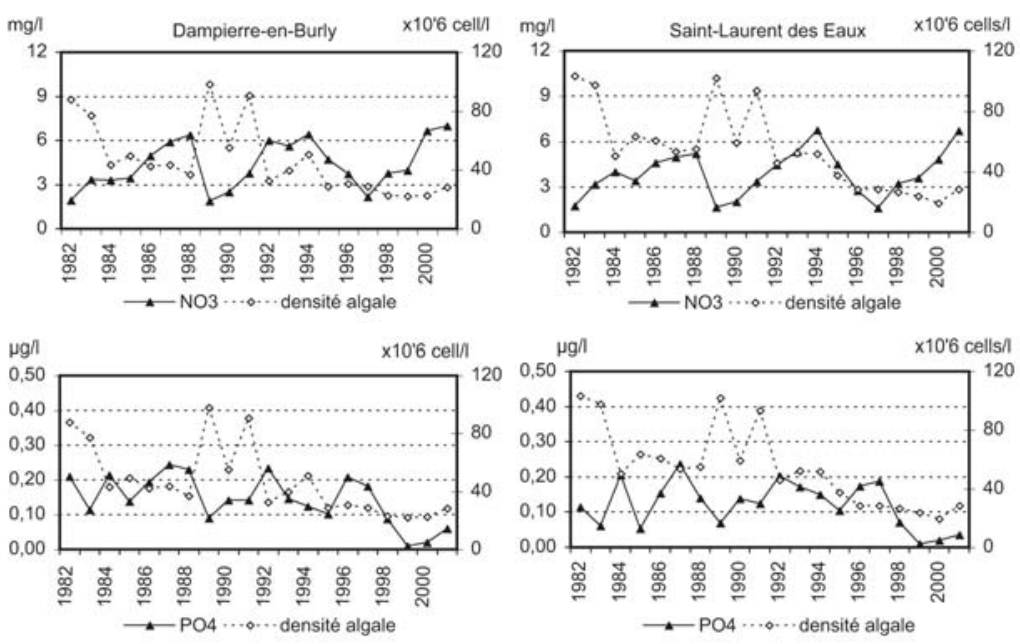

Fig. 5. - Variations des teneurs en éléments nutritifs en relation avec la densité algale $\left(\times 10^{6} \cdot\right.$ cell. $\left.I^{-1}\right)$, pour la période $1982-2001$.

Fig. 5. - Variations in nutrients in relation with the algal density $\left(\times 10^{6} \cdot \mathrm{cell}^{-1} \mathrm{I}^{-1}\right)$, for the period 19822001.

de leur frustules et, bien que les processus métaboliques soient complexes (Rahm et al., 1996 ; Kelly \& Whitton, 1998 ; House et al., 2001) et varient selon les espèces, cet élément peut devenir limitant, d'autant qu'il est aussi utilisé par les communautés benthiques. De telles situations ont été signalées, en particulier en cas de dominance des diatomées Centrales (Lack, 1971 ; Marker \& Collen, 1997), qui sont des algues très abondantes en Loire moyenne. C'est aussi le cas dans certains fleuves comme le Rhin (Admiraal et al., 1993), ou la Seine (Garnier et al., 1995). La $\mathrm{DBO}_{5}$, très liée à la quantité d'algues (corrélations positives hautement significatives), est corrélée négativement aux nitrates, mais elle n'est pas corrélée à $\mathrm{NH} 4$, illustrant la moindre importance des processus de décomposition.

Situation selon l'intensité des étiages

Au regard des variations inter-annuelles de débits, deux types de situations ont été analysées.

Les années 1982 (période 1), 1989-90-91 (période 2) et 1995 à 1999 (période 3), ont été marquées par des étiages particulièrement sévères. Les basses eaux des deux premières périodes sont associées à des maximums algaux, mais ce n'est pas le cas pour la troisième période durant laquelle les algues sont moins denses et l'eau moins oxygénée (Fig. 4). Comparativement aux autres années (dénommées « autres étiages " dans le tableau de corréla- 
tions), pendant ces périodes hydrologiquement déficitaires (dénommées "étiages sévères 》 dans le tableau des corrélations), les teneurs en phosphates ne sont plus corrélées aux débits et à la densité algale, ce qui sous-entend que cet élément devient limitant (hormis les " recyclages rapides ", non détectables par simple analyse chimique). Dans leurs travaux sur l'Elbe, Lehmann \& Rode (2001) indiquent que dans ce cas, les effluents domestiques ou industriels ne jouent pas un rôle majeur dans les apports. Cependant, le ralentissement de la vitesse du courant conduit inévitablement à des réactions en chaîne; une augmentation des surfaces stagnantes favorise le développement des hétérotrophes, voire les échanges entre les sédiments et l'eau dans ces zones très peu profondes, ou encore les recyclages provoqués par le macrobenthos (Essington \& Carpenter, 2000). Ceci amplifie les processus de production là où les recyclages rapides, via les produits excrétés par les animaux, peuvent contribuer aux apports en nutriments. Tandis que la silice n'est plus corrélée aux débits, ces étiages sévères favorisent la production algale. Comparativement à l'ensemble des données ( $d d l=158)$ ou aux années non déficitaires ( $\mathrm{ddl}=$ 86), les corrélations entre $\mathrm{O}_{2}$ et $\mathrm{NH} 4$, entre $\mathrm{O}_{2}$ et $\mathrm{DBO}_{5}$ sont particulièrement fortes durant les années à faible hydraulicité ( $d d l=70)$. Quelques différences apparaissent entre les deux sites: outre des coefficients de corrélations de valeurs différentes
(Tab. II), durant les "étiages sévères », les débits et les algues sont toujours corrélés négativement au niveau du site de Dampierre-en-Burly et la silice est toujours corrélée négativement aux diatomées, ce qui n'est pas observé au niveau du site aval. II est probable que la chute des diatomées observée en 1995 à Saint-Laurent-des-Eaux a été consécutive à un appauvrissement en silice, celui-ci favorisant le développement des Chlorophycées (Gosselain et al., 1994), ce qui fut aussi le cas cette année là. Durant les « autres étiages », les relations $\mathrm{DBO}_{5}-\mathrm{NH} 4$ ne sont plus manifestes en amont, indiquant que les processus de décomposition s'accentuent dans le cours du fleuve. (Fig. 4 ; Tab. II).

\subsubsection{Flux de matière et communautés algales}

$\mathrm{Au}$ regard de la dynamique fluviale, qui est étroitement liée aux débits, les variations des flux d'algues (exprimés en t.j ${ }^{-1}$ ) et de phosphates (exprimés en $\mathrm{kg}^{-j^{-1}}$ ) révèlent, comme pour les nitrates, une forte consommation de cet élément de 1989 à 1991 (Fig. 6) et depuis 1995-1996. Les apports de phosphates étant étroitement liés au lessivage des sols, en période de sècheresse ils peuvent être fortement réduits. Ce fut le cas en 1989-1991 où les flux d'algues avaient sensiblement diminué, mais dans des proportions moindres que ces dernières années (où les débits étaient plus importants que durant la période précédente, cf. Fig. 4). 

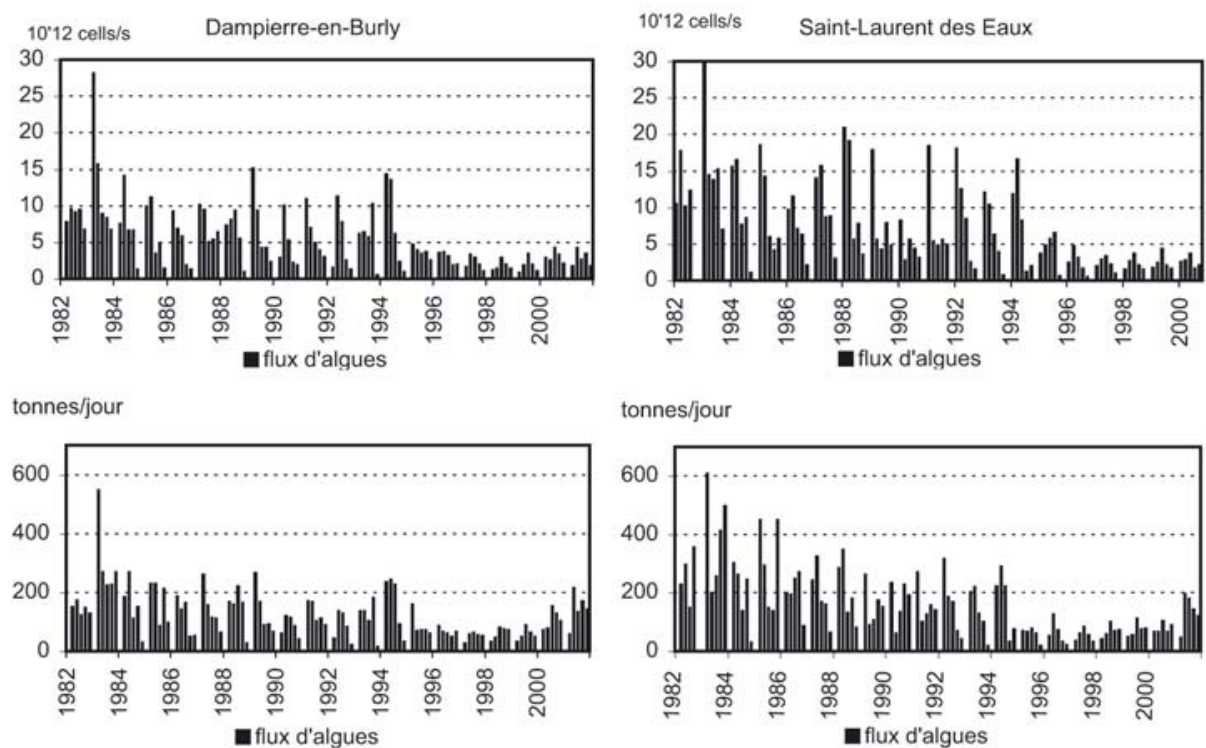

tonnes/jour
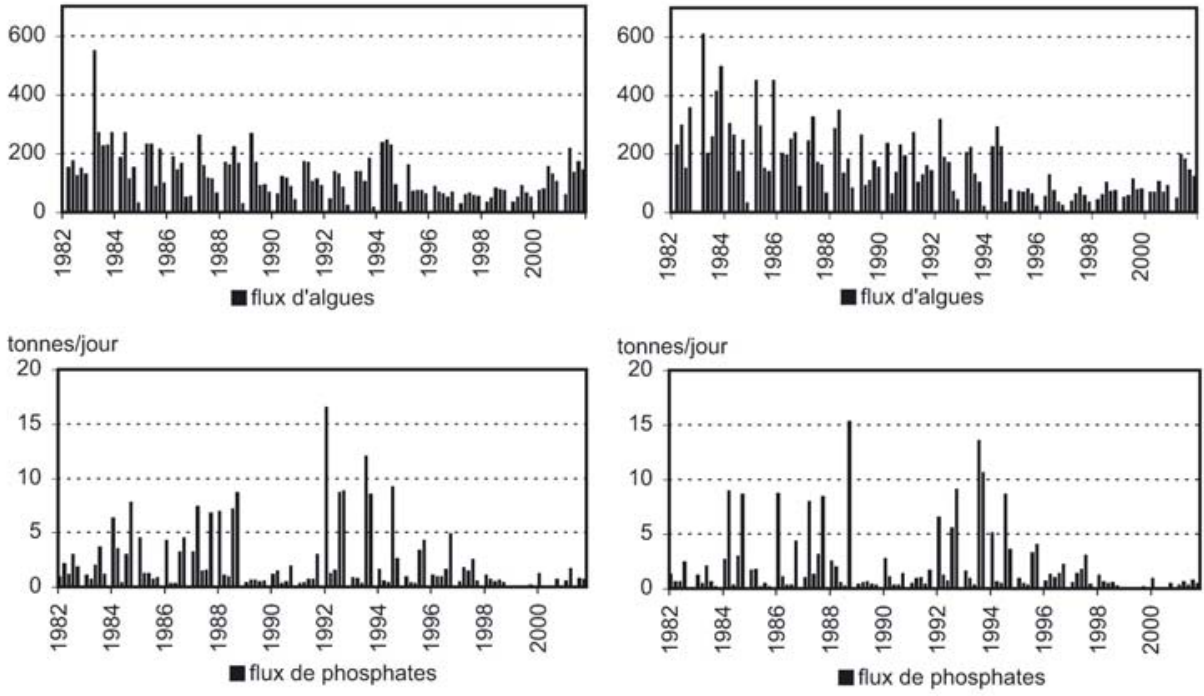

Fig. 6. - Variations des flux d'algues $\left(\times 10^{12} \cdot\right.$ cell. $\left.^{-1}\right)$, exprimés en densités $(\mathrm{N})$ et en biomasses $(\mathrm{B})$, $\left(\mathrm{t}^{-1}\right)$ et des flux de phosphates $\left(\mathrm{t} . \mathrm{j}^{-1}\right)$, pour la période 1982-2001.

Fig. 6. - Variations of the fluxes of algae density $\left(\times 10^{12}\right.$ cell. $\left.^{-1}\right)$, algae biomass $\left(\right.$ t.day $\left.^{-1}\right)$ and phosphates $\left(\right.$ t.day ${ }^{-1}$ ) for the period 1982-2001.

Mais les processus sont complexes, dans la mesure où, par exemple, une augmentation des surfaces stagnantes (Photo 2) peut tout aussi bien favoriser les échanges eau-sédiments et recharger ces zones en phosphates (ne serait-ce que sous l'action des vents thermiques, classiquement observés en Loire par temps sec). A partir de 1994-1995, les flux de phos- phates se sont progressivement abaissés, ainsi que le nombre de cellules algales circulant dans le fleuve.

Globalement, les Cyanobactéries demeurent faiblement représentées en Loire Moyenne où ce sont les Chlorophycées qui dominent largement en densité, suivies des Bacillariophycées (Fig. 7). Et bien que de nombreuses petites diatomées Cen- 

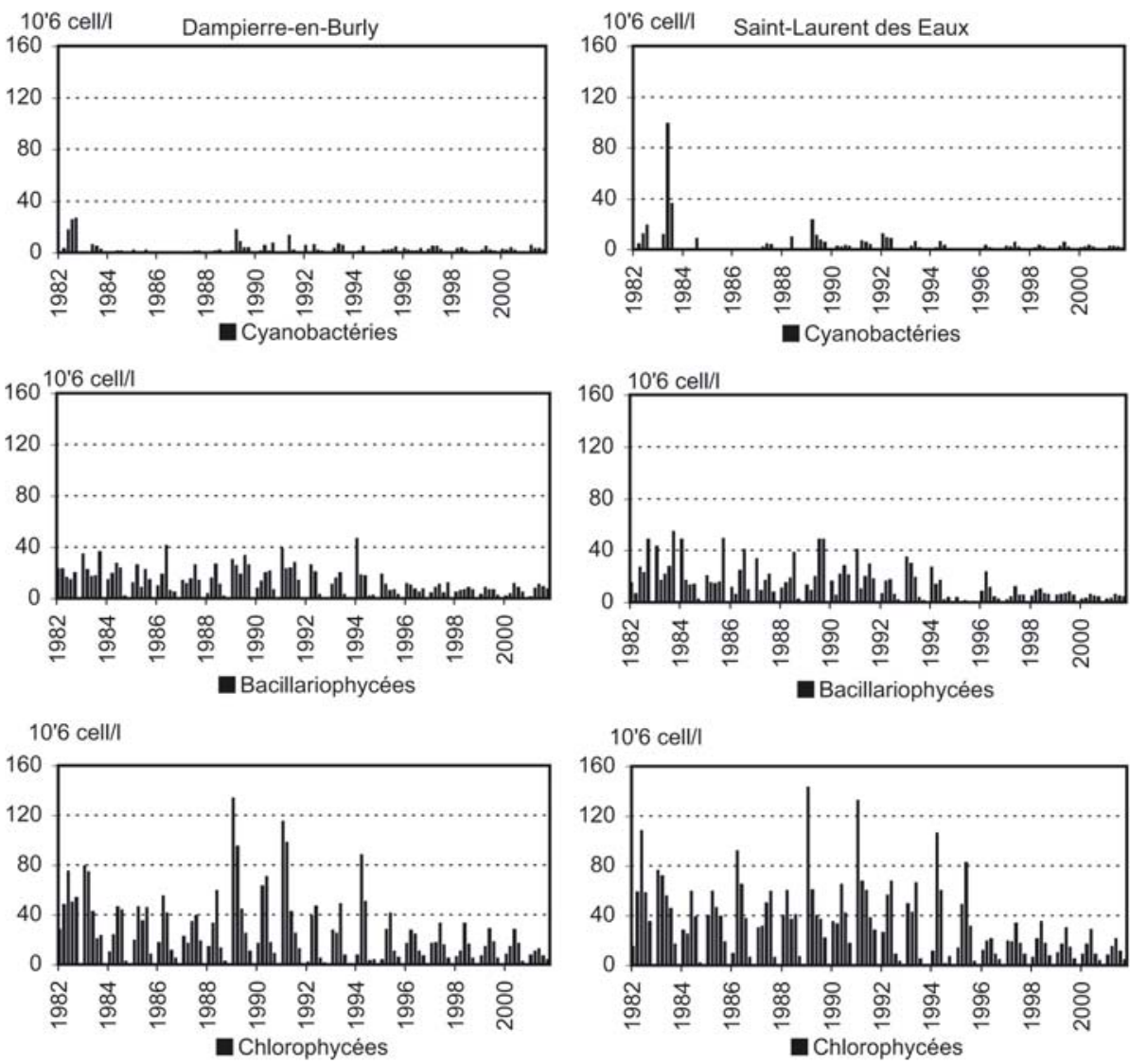

Fig. 7. - Variations de la densité $\left(\times 10^{6}\right.$. cell. $\left.I^{-1}\right)$ des trois principaux groupes d'algues, observées en Loire moyenne pour la période 1982-2001.

Fig. 7. - Variations in density $\left(\times 10^{6}\right.$. cell. $\left.^{-1}\right)$, of the principal algae components, observed in the middle Loire for the period 1982-2001.

trales colonisent régulièrement les eaux du fleuve, ces algues brunes représentent une biomasse plus importante que les algues vertes, dont la majeure partie des cellules est de petite taille (Lair et al. 1996; Lair \& Reyes-Marchant, 2000).

Ces 20 années d'observation permettent de constater qu'en période estivale, l'oxygénation de l'eau est très liée à l'activité photosynthétique, la baisse de densité algale observée au cours de ces dernières années étant concomitante à celle des teneurs en oxygène dissous. Bien qu'aucun système indicateur basé sur le couple biomasse algale - éléments nutritifs n'ait été appliqué avec succès (Weir \& Descy, 1998), cette situation implique qu'une baisse de la 
quantité d'algues peut entraîner une baisse de la qualité de l'eau (Descy, 1992), ce qui soulève un problème de gestion. Les phosphates exercent probablement un contrôle ascendant et, bien qu'il y ait besoin d'un plus grand recul pour le confirmer, il est fort probable que les efforts d'épuration développés par l'Agence de l'Eau Loire-Bretagne sont en train de porter leurs fruits. Mais les algues peuvent aussi être régulées par leurs consommateurs, qui exerceraient alors un contrôle descendant. C'est pour tenter de répondre à cette question que la distribution des rotifères, constituants quasi-exclusifs du potamoplancton, est étudiée de manière suivie depuis 1995.

\subsection{Deuxième regard : le moyen terme}

\subsubsection{Rôle des algues dans} l'oxygénation de l'eau

La période 1995-2001, durant laquelle les rotifères ont été étudiés, est caractérisée par des débits faibles (oscillant autour de $100 \mathrm{~m}^{3} . \mathrm{s}^{-1}$ ), par quelques épisodes pluvieux en 1995, 2000 et 2001 et, en plein été, par des températures et des $\mathrm{pH}$ élevés (Fig. 8). Les teneurs en nitrates, plus fortes en 2000 et 2001 (Fig. 5), sont réglées par les variations de débits (les corrélations positives sont hautement significatives), (Tab. III). Comparativement aux années d' « étiages sévères ", examinées précédem-
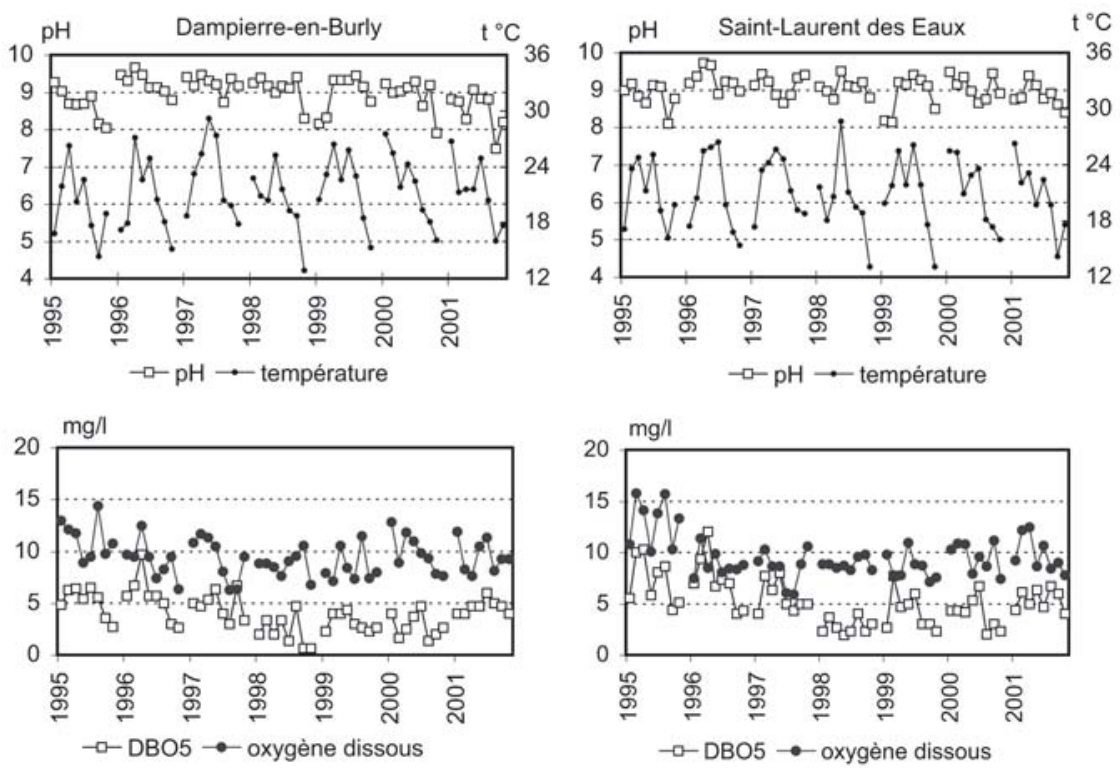

Fig. 8. - Variations du pH et de la température, de la $\mathrm{DBO}_{5}$ et des teneurs en oxygène dissous, observées en Loire moyenne pour la période 1995-2001.

Fig. 8. - Variations in $\mathrm{pH}$ and temperature, $\mathrm{BOD}_{5}$ and dissolved oxygen, observed in the middle Loire for the period 1995-2001. 
ment, la $\mathrm{DBO}_{5}$ n'est plus corrélée à $\mathrm{NH} 4$ et les teneurs en oxygène dissous sont corrélées à cette forme d'azote seulement à Saint-Laurentdes-Eaux, suggérant que les processus de nitrification sont moins rapides en amont. Les quantités de phosphates, qui sont très faibles (le plus souvent $\left.<0,20 \mu \mathrm{g} \cdot \mathrm{I}^{-1}\right)$, ne sont pas corrélées aux débits, une situation rencontrée durant les périodes d' "étiages sévères ", et les teneurs en $\mathrm{SiO}_{2}$ qui se sont abaissées au cours des étiages plus marqués des années 1997 à 1999 ne sont plus corrélées aux débits à Saint-Laurentdes-Eaux (Tab. III). Dans ce secteur aval, la densité des diatomées avait été particulièrement faible en 1995 , contrairement à celle des algues vertes (Fig. 7).

Les débits sont toujours corrélés négativement à la quantité d'algues. Les teneurs en oxygène dissous, qui sont légèrement inférieures en aval (sauf à Saint-Laurent-des-Eaux en 1995 où la densité d'algues était plus importante), sont fortes en début de saison et chutent en fin d'été, les remontées de fin septembre - début octobre étant liées aux débits. Durant cette période, et quel que soit le site, il n'y a plus de corrélation entre la densité algale et la teneur en oxygène dissous, par contre les corrélations entre la densité algale et la demande biochimique en oxygène sont positives et hautement significatives (Tab. III). Bien que plus forte et plus contrastée sur le site aval, comparativement au site amont (Fig. 8), la $\mathrm{DBO}_{5}$ a diminué sur les deux sites
Tableau III. - Corrélations entre variables, calculées à partir des données recueillies aux trois stations de chaque site durant la période 1995-2001.

Table III. - Correlation between variables, extracted from the data collected at each station and site, for the period 1995-2001.

\begin{tabular}{|c|c|c|}
\hline Variables corrélées & Dam & SIb \\
\hline débits /NO3 & 0,74 & 0,72 \\
\hline débits /PO4 & $\#$ & $\#$ \\
\hline débits /SiO2 & 0,40 & \# \\
\hline débits / $\mathrm{N}$ algues & $-0,55$ & $-0,50$ \\
\hline O2/débits & $\#$ & $\#$ \\
\hline O2/NO3 & \# & \# \\
\hline $\mathrm{O} 2 / \mathrm{NH}_{4}$ & \# & $-0,37$ \\
\hline $\mathrm{O} 2 / \mathrm{N} \& \mathrm{~B}$ algues & $\#$ & $\#$ \\
\hline DBO5/N algues & 0,49 & 0,48 \\
\hline $\mathrm{DBO} 5 / \mathrm{O} 2$ & 0,36 & 0,39 \\
\hline $\mathrm{DBO} / \mathrm{NO} 3$ & $-0,39$ & $\#$ \\
\hline $\mathrm{DBO} 5 / \mathrm{NH} 4$ & $\#$ & \# \\
\hline DBO5/N:P & $-0,37$ & $-0,36$ \\
\hline DBO5/B algues & $\#$ & $\#$ \\
\hline $\mathrm{NO} 3 / \mathrm{N}$ algues & $-0,61$ & $-0,51$ \\
\hline $\mathrm{PO} 4 / \mathrm{N}$ algues & $\#$ & $\#$ \\
\hline SiO2/N diatomées & $\#$ & \# \\
\hline débits/N rotifères & $-0,40$ & $\#$ \\
\hline$N$ algues/N rotifères & 0,74 & 0,63 \\
\hline$B$ algues/N rotifères & 0,43 & 0,41 \\
\hline $\mathrm{N}$ chlorophycées/N rotifères & 0,73 & 0,65 \\
\hline $\mathrm{N}$ diatomées/N rotifères & \# & \# \\
\hline
\end{tabular}

jusqu'en 2000 (avec des valeurs $<5 \mathrm{mg} \cdot l^{-1}$ à Dampierre-en-Burly, un chiffre rarement dépassé à SaintLaurent-des-Eaux.) ; elle n'est plus corrélée à la biomasse algale (qui peut agir comme facteur de "pourrissement"), suggérant qu'il y avait moins de substrat organique pour soutenir l'activité bactérienne, voire moins d'algues actives (cette $\mathrm{DBO}_{5}$ est remontée en 2001). Les variations du rapport acide (lié aux dosages de chlorophylle) sont peu marquées d'une année sur l'autre; ses valeurs oscillent le plus souvent entre 1,0 et 1,4 , révélant des algues 
en mauvais état physiologique ; les valeurs $>1,7$ (bon état) et ponctuellement $>2$ (fin juin 1998 à Dampierreen-Burly) ou > 3 (début août 1998 à Saint-Laurent-des-Eaux), sont exceptionnelles. Enfin, si l'intensité lumineuse est une autre variable impliquée dans les processus photosynthétiques, la profondeur de disparition du disque de Secchi est du même ordre de grandeur comparativement aux années antérieures à 1995. Elle varie peu d'une saison à l'autre et oscille autour de $55-60 \mathrm{~cm}$, atteignant exceptionnellement le mètre, comme ce fut le cas en octobre 2000 sur les deux sites (ce qui n'exclut pas que l'auto-ombrage puisse limiter la production).
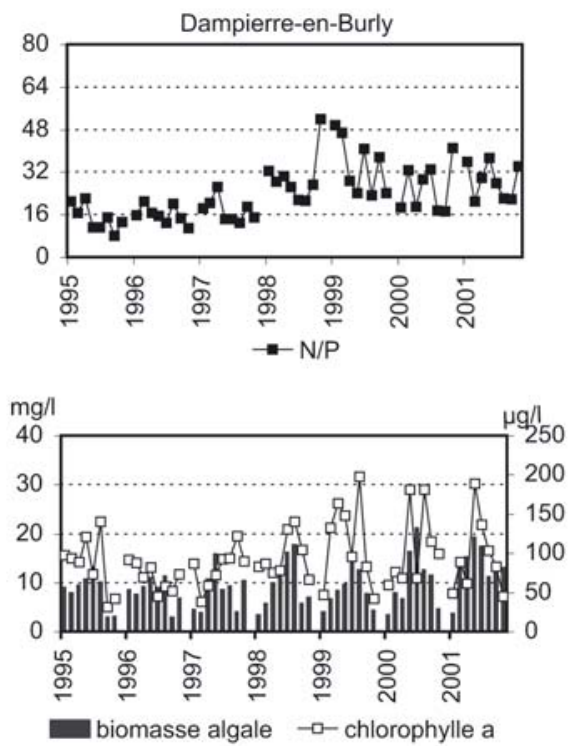

\subsubsection{Une limitation par les} nutriments?

Sur la période 1995-2001, la densité algale est corrélée négativement aux nitrates, mais pas aux phosphates qui pourraient être limitants. De 1995 à 1997, le rapport N/P est resté voisin de 16 (en référence à l'indice de Redfield = $16: 1$ ), (Goldman, 1980), indiquant une large consommation de nitrates (et une $\mathrm{DBO}_{5}$ plus forte). II est remonté nettement au-dessus de cette valeur à partir de 1998 (Fig. 9). L'augmentation de ce rapport N/P est concomitante à une relative augmentation des quantités de Cyanobactéries. Il a été montré en mésocosmes (avec fertilisants + apports naturels) que des Cyanobactéries sans hétéro-
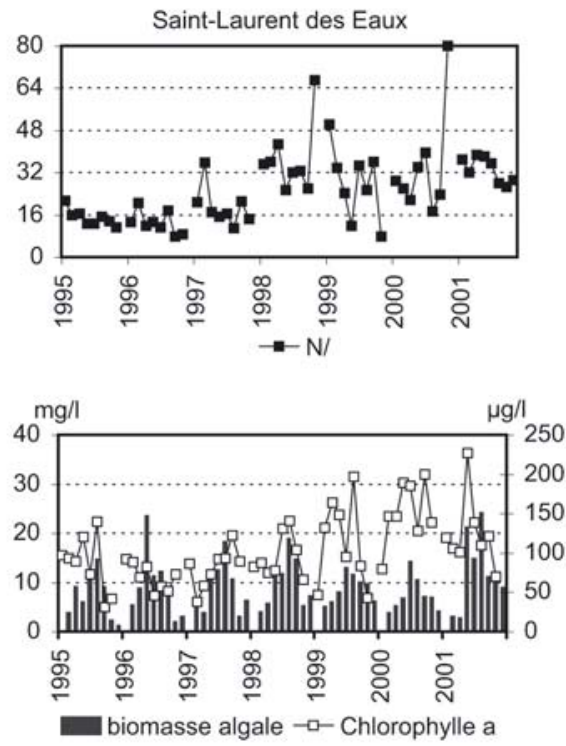

Fig. 9. - Variations du rapport N/P, ainsi que de la biomasse algale, durant la période 1995-2001.

Fig. 9. - Variations in N/P and algal biomass, for the period 1995-2001. 
cystes peuvent devenir dominantes avec des rapports supérieurs à $17: 1$ (Levine \& Schindler, 1999). Ces algues bleues sont favorisées par des environnements physiquement stables (Pearl, 1988) ; en Loire, elles peuvent simplement provenir de situations locales, liées à des conditions hydrologiques particulières, d'autant que les fluctuations des teneurs en éléments nutritifs (cas des zones peu profondes) exercent un rôle déterminant dans la composition des communautés phytoplanctoniques et influencent l'issue des compétitions interspécifiques.

Durant cette période 1995-2001, et contrairement à la densité, la biomasse algale tend à augmenter, ce qu'indiquent également les teneurs en chlorophylle a (Fig. 9). L'observation des variations de densité des principaux groupes algaux confirme la présence de très petites quantités de Cyanobactéries, d'autant que comparativement au long terme, leur augmentation est insignifiante, des densités plus importantes ayant été mesurées antérieurement à plusieurs reprises (Fig. 7). Comme nous l'avons souligné précédemment, une baisse progressive de la densité (et de la biomasse) des Chlorophycées se dessine ; ces algues sont une source de nourriture pour les filtreurs qui prolifèrent en étiage dans le lit du fleuve, en particulier dans les zones de courant faible à nul (Picard, Doctorat en préparation). A l'exception de 1995 (année particulièrement pauvre en diatomées), la densité des Bacillariophycées demeure assez stable (Fig. 10). La distribution des biomas- ses révèle un certain changement de composition du peuplement, puisqu'il s'est produit un développement progressif de Cyanobactéries du genre Oscillatoria sur les deux sites. La biomasse des Chlorophycées demeure constante sur l'ensemble de cette période à Dampierre-en-Burly et depuis 1996 à Saint-Laurent-des-Eaux. A l'exception d'un minimum à SaintLaurent-des-Eaux en 1995 (année dominée par les Chlorophycées) et d'un maximum à Dampierre-en-Burly en 2001 (dû à une prolifération de $C y$ clotella meneghiniana), globalement la biomasse des Bacillariophycées tend à augmenter au niveau de Dampierre-en-Burly ; elle fluctue davantage à Saint-Laurent-des-Eaux (Fig. 10). La teneur en silice n'est pas corrélée aux diatomées, comme c'était le cas à Saint-Laurent-desEaux durant les "étiages sévères ", suggérant alors un certain épuisement de cet élément.

\subsubsection{Approche du fonctionnement écotrophique}

II est certain qu'à teneurs en chlorophylle a comparables, les métazoaires planctoniques (réduits en Loire moyenne aux rotifères) sont moins denses en eau courante, par rapport aux lacs. Les fortes densités de rotifères rencontrées dans certaines rivières sont associées aux zones d'eaux mortes (Lair \& ReyesMarchant, 1997 ; Galkovskaya \& Molotkov, 2001). Généralement, les rotifères sont favorisés par les basses eaux et de ce fait corrélés négativement aux débits (Kobayashi et al., 

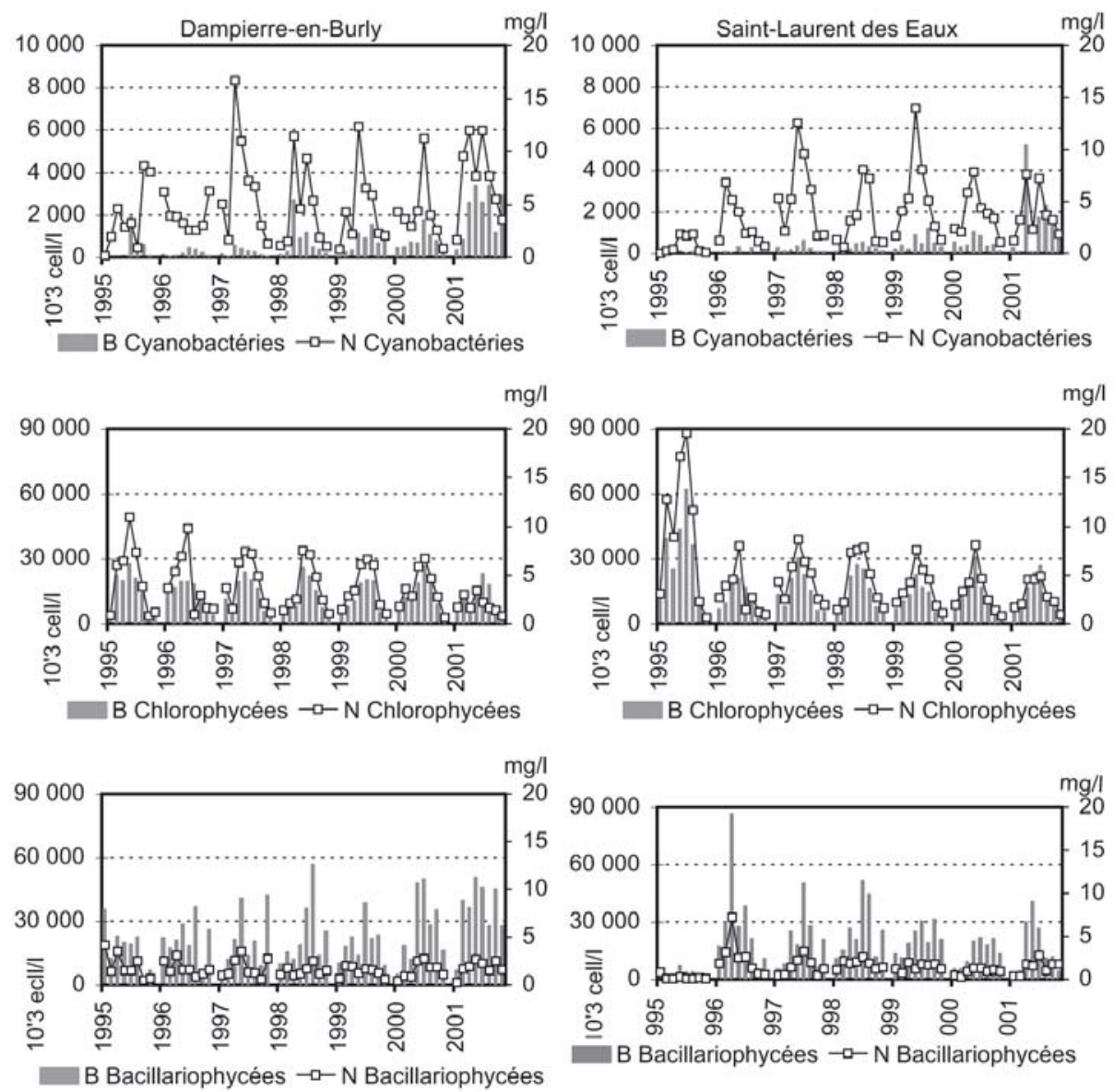

Fig. 10. - Comparaison des variations de densité (N) et de biomasse (B) des trois principaux groupes d'algues, rencontrés en Loire moyenne durant la période 1995-2001.

Fig. 10. - Comparison in the density $(\mathrm{N})$ and biomass $(\mathrm{B})$ variations of the three principal algal components observed in the middle Loire, for the period 1995-2001.

1998) et, dans ce secteur de Loire, leur densité moyenne fluctue d'un site à l'autre (Fig. 11). Cependant, la densité mesurée à chacune des sorties est corrélée négativement aux débits à Dampierre-en-Burly seulement (Tab. III). Ces organismes sont étroitement liés à la densité (et à la biomasse) algale, à l'exception de 1999 à Dampierre-en-Burly où le maximum algal n'a pas été accompagné d'une prolifération de rotifères (Fig. 11), (et nous en examinerons la cause avec les données de base). A l'échelle bi-mensuelle, leurs maximums de densité sont observés si- 

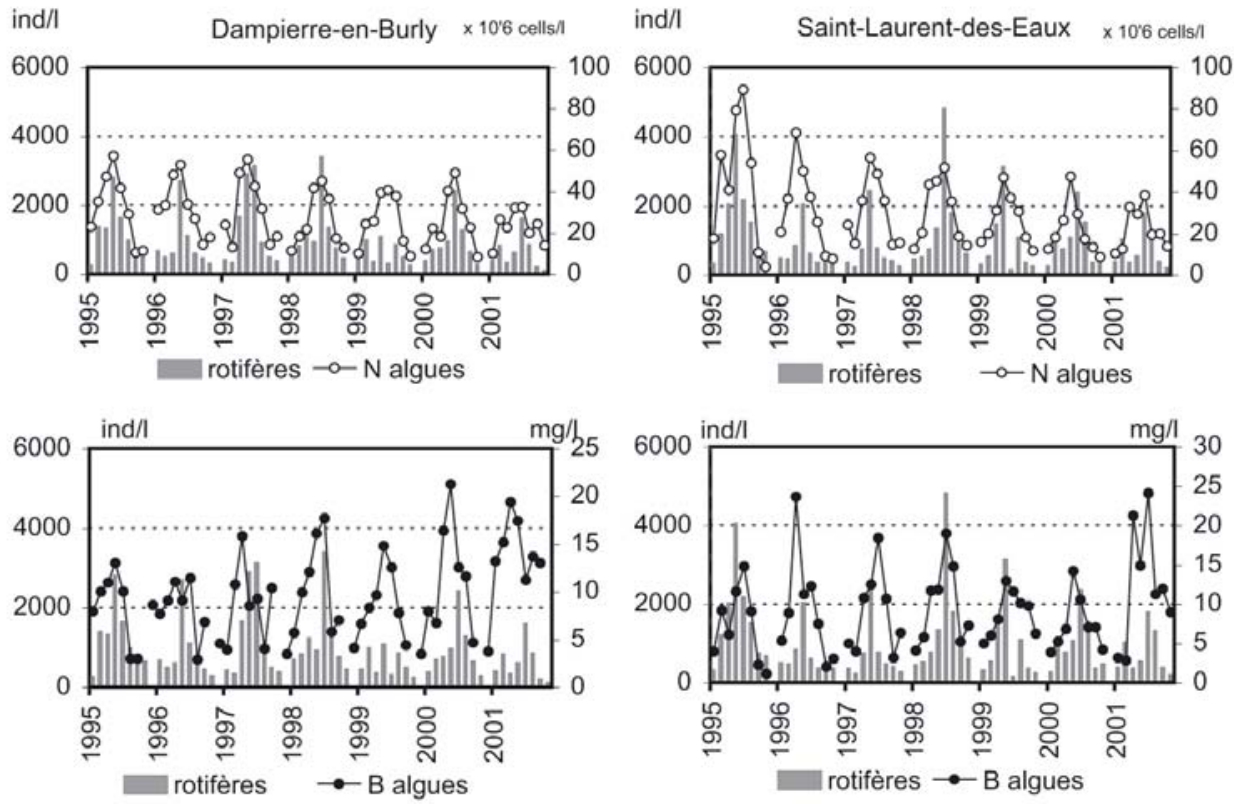

Fig. 11. - Distribution de la densité $(N)$ et de la biomasse $(B)$ des algues comparée aux variations de densité des rotifères, durant la période 1995-2001.

Fig. 11. - Distribution of the density $(\mathrm{N})$ and biomass $(\mathrm{B})$ of the algae in comparison with the rotifers density for the period 1995-2001.

multanément aux maximums algaux, ou deux semaines plus tard, et leur régression est parallèle à celle des algues.

Les corrélations positives, hautement significatives entre la densité (et la biomasse) algale et la densité des rotifères (Tab. III), suggèrent que la nourriture algale (qui n'est cependant pas la seule nourriture consommable par ce type d'organismes), n'est pas limitante. La consommation des rotifères s'exerce sur les Chlorophycées qui sont globalement de taille plus petite que les diatomées, comme le montrent les corrélations positives hautement significatives avec ce groupe d'algues et l'absence de corrélations avec les diatomées (Tab. III). Nous pouvons en déduire que les rotifères chutent en même temps que ces algues et qu'ils sont susceptibles de les réguler, mais sont-ils la cause essentielle de leur diminution? sachant que ces organismes ont également à leur disposition tout un cortège d'unicellulaires hétérotrophes pour se nourrir (Lair et al., 1998, 1999 ; Picard, en préparation).

Les résultats des calculs de la diversité spécifique (Shannon \& Weaver, 1949), qui exprime le degré d'organisation des peuplements, montrent que 
les communautés végétales (microalgues) et animales (rotifères) du potamoplancton sont, dans l'ensemble, bien diversifiées (Fig. 12). La diversité spécifique algale était plus faible en 1995-1996, comparativement aux années suivantes où elle est demeurée assez stable. Au regard du nombre d'espèces (de l'ordre de 180) couramment rencontrées dans ce secteur de fleuve (Lair \& Reyes-Marchant, 2000), la diversité spécifique algale est généralement supérieure à celle des rotifères, dont les représentants sont moins nombreux (de l'ordre de 50 espèces). Ceux-ci sont bien diversifiés à Dampierre-en-Burly jusqu'en 1999 ( \pm 4 bits par individu). A partir de cette date, la cause de leur déstructuration est indépendante du fonctionnement de la centrale; elle est liée à la présence, en fortes quantités, de rotifères prédateurs du genre Asplanchna. A Saint-Laurent-desEaux, comme nous l'évoquerons plus en détail ci-après, l'installation du diffuseur en 1996 a perturbé les rotifères filtreurs, en raison de la présence de matériel en suspension lié aux travaux d'excavation. L'année suivante, ces organismes étaient à nouveau bien structurés, et leur diversité spécifique est demeurée stable jusqu'en fin d'étude.

Durant la période 1995-2001, la réduction de la quantité de phosphates a conduit à une diminution de la quantité d'algues. Consécutivement, les teneurs en oxygène dissous ont baissé à partir de 1995 à Dampierre-enBurly et à partir de 1996 à Saint-Laurent-des-Eaux, ce qui signifie que la production primaire a un effet moins important sur la balance en oxygène, comparativement aux années précédentes, induisant un rôle plus important de la part des hétérotrophes. D'un point de vue fonctionnel, les études actuellement en cours sur le potamoplancton, dont les bactéries, devraient permettre de mieux comprendre l'organisation fonctionnelle du réseau trophique et - en particulier - l'intrication des processus d'autotrophie et d'hétérotrophie (Picard, en préparation).
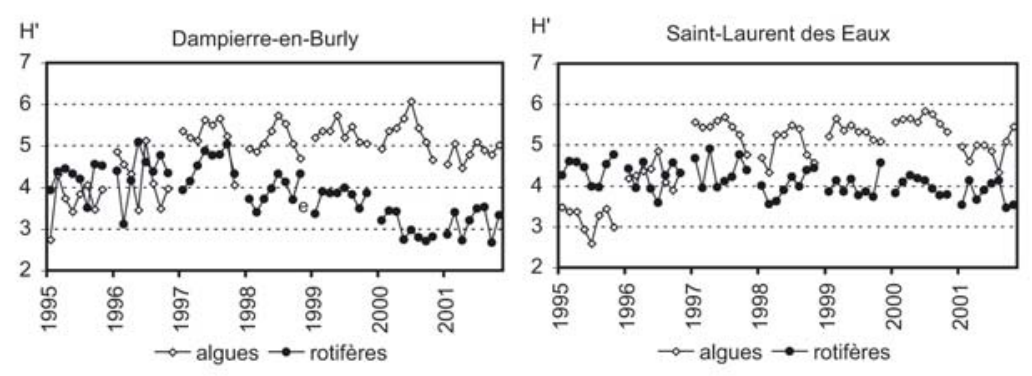

Fig. 12. - Variations de la diversité spécifique $\left(\mathrm{H}^{\prime}\right)$ des algues et des rotifères, durant la période 1995-2001.

Fig. 12. - Variations in the specific diversity of algae and rotifers, for the period 1995-2001. 


\subsection{Troisième regard : les données de base.}

Au regard de l'hétérogénéité de ce type de milieu, le choix des échelles d'observation demeure un élément crucial (Hildrew, 1996 ; Swan \& Palmer, 2000), ce qui nous a conduit à revenir aux données de base, de manière à analyser les processus locaux qui sont étroitement liés à la dynamique hydrologique (Tockner et al., 1999). Le but de ces études, rappelons-le, était de contrôler la qualité des eaux en amont des centrales nucléaires de production d'électricité, de mesurer leur impact en aval et, en particulier, celui des rejets d'eaux chaudes. La synthèse des données recueillies de 1977 à 1998 sur le site de Saint-Laurent-des-Eaux a montré le resserrement des écarts de température entre stations, par suite de l'arrêt successif des deux tranches de la filière à uranium naturel graphite gaz (UNGG) de Saint-Laurent A en 1990 et 1992 et de l'installation d'un diffuseur en 1996 (Lair \& Reyes-Marchant, 2000). Ce type d'ouvrage a pour but de répartir, sur toute la largeur du fleuve, le rejet d'eaux réchauffées sortant de la centrale, afin d'obtenir un effet de dilution initiale plus rapide.

Les enregistrements horaires des stations automatiques installées par EDF sont les témoins des variations journalières de température; or, sur chaque site, les séries de prélèvements et de mesures nécessaires aux «suivis hydroécologiques" s'étalent sur plusieurs heures. A chaque sortie, la station amont est analysée en premier et la station aval b en dernier, ce qui induit inévitablement des écarts de température, liés au réchauffement diurne, lui-même fonction des conditions climatiques; Wu et al. (2001) ont souligné les difficultés d'analyse de ce type de variations. Cependant, les données recueillies pour la période 1995-2001 montrent que les écarts de température entre la station amont et celle qui est située en aval immédiat du rejet (aval a) sont très faibles, ce que confirment les courbes de tendances (Fig. 13). L'écart de température de l'eau le plus important (variations naturelles liées à l'hétérogénéité du milieu + échauffement thermique résiduel) a été de $4^{\circ} \mathrm{C}$, pour des mesures effectuées par temps calme et ensoleillé le 24 août 1999 à Dampierre-en-Burly, l'une à 12 h 40 en amont et l'autre à $16 \mathrm{~h}$ en aval b (station la plus éloignée où la dilution a déjà eu lieu). En outre, dans cette zone de méandres, selon les conditions hydrologiques du moment (Photos 1 \& 2), la température des eaux peut être la résultante d'un mélange entre l'eau du chenal principal et celle de zones peu profondes qui se forment à l'étiage sur de larges surfaces occupant la largeur du fleuve. Ces dernières sont inévitablement plus chaudes et, à l'évidence, l'eau qui coule ne présente pas toujours une température naturelle uniforme, en raison de l'hétérogénéité naturelle du milieu. Cette situation conduit à des écarts de température conséquents, comme ce fut observé au cours d'une 

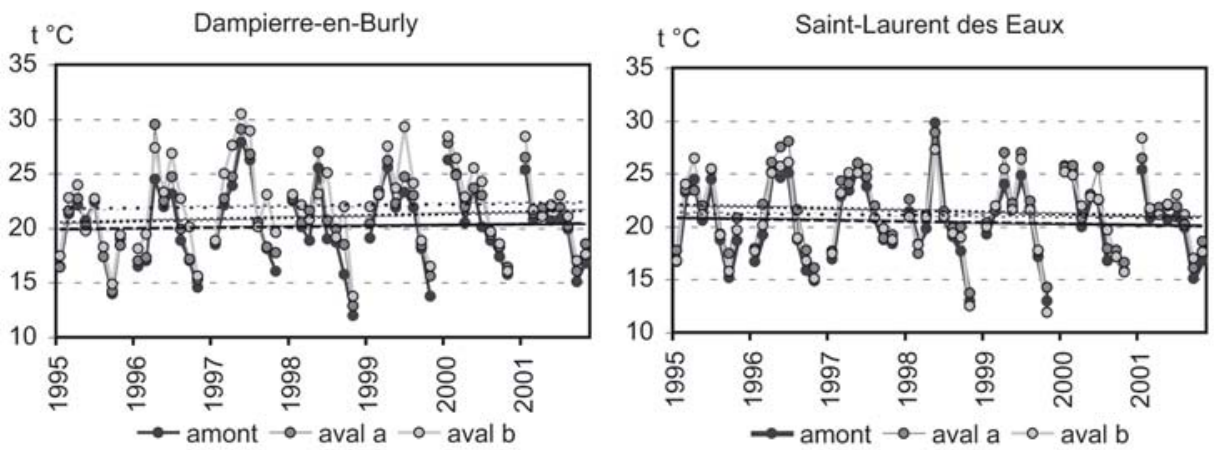

Fig. 13. - Variations de la température des eaux de la Loire Moyenne à chacune des trois stations échantillonnées sur chaque site, durant la période 1995-2001.

Fig. 13. - Variations in the water temperature of the middle Loire at each of the three sampling stations, for the period 1995-2001 (upstream, immediate downstream and distant downstream).

analyse d'un secteur du fleuve réalisée au niveau de Muides-sur-Loire au cours de l'été 2000 (Picard, Lair \& Reyes-Marchant, en préparation).

\subsubsection{Des communautés bien adaptées à leur milieu}

L'examen des densités d'algues et de rotifères mesurées à chacune des stations de 1995 à 2001 révèle : (1) une tendance générale à la diminution de la quantité d'algues (voire de rotifères) (Fig. 14). (2) A Dampierreen-Burly, les courbes linéaires de tendance, tracées pour les algues à chacune des 3 stations, sont très proches (à superposées). Sur ce même site, la courbe linéaire "rotifères-aval b" (station plus densément peuplée assez régulièrement), qui a une pente plus accentuée, "décroche". (3) A Saint-Laurent-des-Eaux, les courbes linéaires des deux communautés sont décrochées en aval $b$, un sec- teur où la densité des algues était nettement supérieure en 1995 et 1997. Cette station est située à plusieurs kilomètres des rejets, et leur possible influence a été gommée par effet de dilution. Sur ce site, comme sur le précédent, la densité des rotifères est supérieure en aval assez régulièrement. Bien que ces organismes parthénogénétiques soient capables de se multiplier dans le courant, les augmentations de densité observées régulièrement à quelques kilomètres en aval ne peuvent provenir, à l'évidence, que d'ensemencements locaux (Lair \& Reyes-Marchant, 1997 ; Picard, en préparation). De tels résultats soulignent le rôle de la morphologie du lit du fleuve dans les processus biologiques. Cependant, ces différences locales de densité ne gomment pas l'effet « continuum fluvial » qui s'exprime par des variations globales de densité très proches de Dampierre-en-Burly à Saint-Laurentdes-Eaux, pourtant distants de $90 \mathrm{~km}$, 


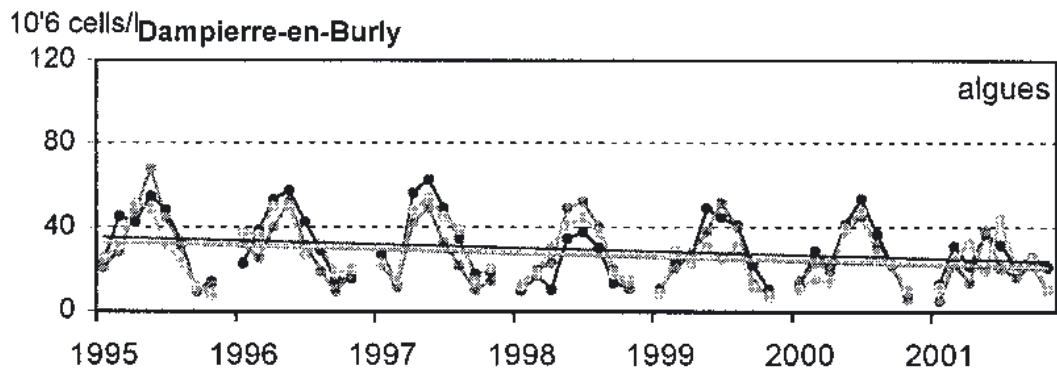

$10^{\prime} 6 \mathrm{ce}|\mathbf{s} /|$
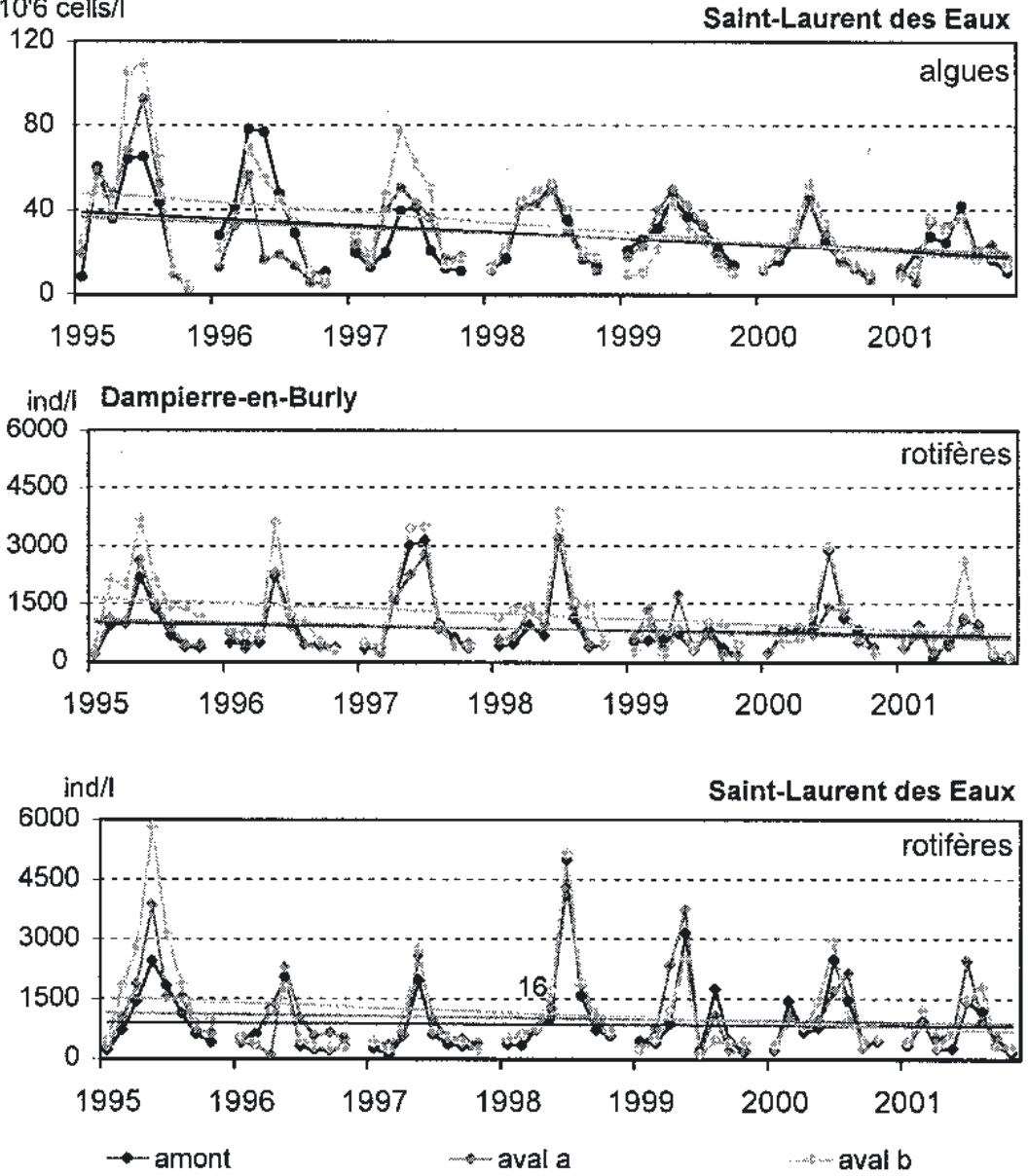

Fig. 14. - Variations de la densité des algues et des rotifères à chacune des trois stations échantillonnées sur chaque site, durant la période 1995-2001.

Fig. 14. - Variations in the algae and rotifers density at each of the three sampling stations, for the period 1995-2001. 
ce qui n'exclut pas d'importantes variations spécifiques entre sites.

\subsubsection{Un milieu résilient}

Durant la période 1996-2001, divers travaux ont été effectués dans le fleuve, susceptibles d'affecter la qualité des eaux et, grâce aux "suivis écologiques » bi-mensuels, l'examen détaillé des différents paramètres physiques, chimiques et biologiques, analysés au cours des "suivis écologiques " annuels, a permis de mettre en relation les évènements survenus sur le cours du fleuve et la réponse des organismes.

\section{Effet des chlorations}

\section{sur les communautés vivantes}

Afin de nettoyer les réfrigérants atmosphériques du site de Dampierre, suite à la prolifération de salissures biologiques (amibes pathogènes), une chloration "purge fermée » puis en continu «purge ouverte » a été réalisée du 29 juin au 7 juillet 1996, tout en respectant la concentration de $0,1 \mathrm{mg} \cdot \mathrm{l}^{-1}$ en chlore libre, autorisée dans les eaux potables et dans les rejets (EDF, Direction des Etudes et Recherches, 1996). La résultante d'une chloration (qui, à l'extrême, peut conduire à une stérilisation du milieu biologique), peut se traduire par la disparition des espèces les plus sensibles. C'est ainsi qu'une baisse significative de la richesse spécifique des algues planctoniques et des rotifères (ainsi que des diatomées benthiques déposées sur les supports artificiels) est observée aux deux stations aval le 8 juillet (cam- pagne 01), lendemain de l'arrêt des chlorations, à l'occasion de la sortie bi-mensuelle du "suivi écologique" (Fig. 15). Cependant, dès la seconde campagne de juillet ( $\mathrm{N}^{\circ} 2$ le 23$)$, une remontée de la richesse spécifique algale est constatée aux deux stations et, le 19 août, elle atteint des valeurs comparables aux trois stations. Quant à la richesse spécifique des rotifères (faible aux deux stations aval le 8 juillet), dès le 23 juillet elle atteint rapidement des valeurs maximales en aval éloigné et, au début du mois d'août, elle est forte aux trois stations. Ces résultats attestent de la rapide récupération du milieu par ces organismes vivants.
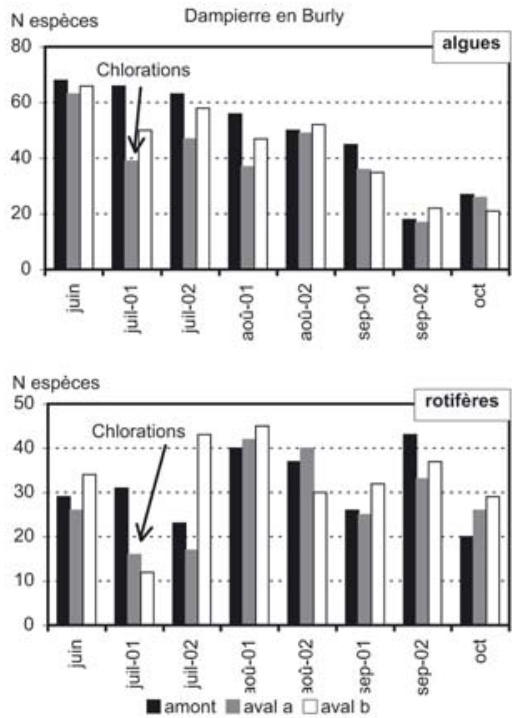

Fig. 15. - Effets des chlorations effectuées en 1996 à Dampierre-en-Burly, sur la richesse spécifique des algues et des rotifères.

Fig. 15. - Effect of the chlorinations performed in 1996 at Dampierre-en-Burly on the specific richness of algae and rotifers. 
Quantitativement, la densité algale a légèrement chuté en aval (Fig. 14) ; cette baisse est liée à celle des Bacillariophycées, des algues plus sensibles que les autres groupes aux variations de la qualité des eaux. La densité des rotifères est demeurée proche aux stations amont et aval immédiat ; en fait, un examen plus précis des communautés montre qu'il s'est produit, en aval, une baisse de densité des Trichocercidae, une famille de rotifères plus sensible que les autres aux variations de la qualité des eaux. En conséquence, la structure des rotifères (exprimée par leur diversité spécifique) a été plus affectée que celle des algues (Fig. 12), dans la mesure où l'influence des chlorations s'est traduite par une perte temporaire d'espèces particulièrement polluo-sensibles. Mais cela confirme bien que le milieu a rapidement récupéré.

\section{Effet des travaux de terrassement sur les communautés vivantes}

Sur le site aval de Saint-Laurentdes-Eaux, un diffuseur a été installé en 1996. La même année, des travaux d'amélioration de la passe à poissons ont également été effectués. De plus, à partir de 1997, les travaux de restauration des piles du vieux pont de Beaugency, situé à une dizaine de kilomètres en amont de Saint-Laurent-des-Eaux, ont provoqué l'arrivée de grandes quantités de MES au niveau du CNPE (un maximum $>500 \mathrm{mg} . \mathrm{l}-1$ a été mesuré le 9 juillet en amont). Sur le site amont de Dampierre-en-Burly, les travaux de terrassement réalisés en 1999 sur la passe à poissons existante et pour aménager une passe à canoëscayaks, ont été emportés par une forte crue durant l'hiver 1999-2000 et ont dû être refaits.

La mise en suspension de telles quantités de matériaux (dont plusieurs tonnes de sédiments), induit des modifications plus complexes que les chlorations. II peut se produire une baisse locale et momentanée de la transparence (et une réduction de la photosynthèse), un colmatage des fonds (qui peut durer des années si les hautes eaux n'assurent pas un balayage général) et la présence de matières en suspension représente une gêne pour les organismes filtreurs de particules. À l'inverse, le remuage des fonds contribue à la remise en circulation de divers composés (dont le phosphore, avec ses conséquences sur la production algale), à une amplification des activités bactériennes, avec leurs conséquences à la fois sur l'oxygénation de l'eau, sur le développement des organismes consommateurs de bactéries (dont des protozoaires flagellés et ciliés, sources de nourriture pour les métazoaires), etc.

Nous prendrons l'exemple de Saint-Laurent-des-Eaux.

En 1996, les teneurs en oxygène dissous sont demeurées inférieures aux stations situées en aval du CNPE, tandis que les matières en suspension augmentaient, ainsi que la demande en oxygène, un phénomène manifeste à partir de la deuxième quinzaine d'août 1996 
(Fig. 16). L'augmentation des débits en fin d'étude a permis de réoxygéner le milieu, mais les MES sont demeurées. En 1997, l'arrivée de MES venues du secteur de Beaugency a provoqué une baisse des teneurs en oxygène dissous et une augmentation ponctuelle de la $\mathrm{DBO}_{5}$ en amont du CNPE; mais ces nuisances se sont peu répercutées en aval, en rai-
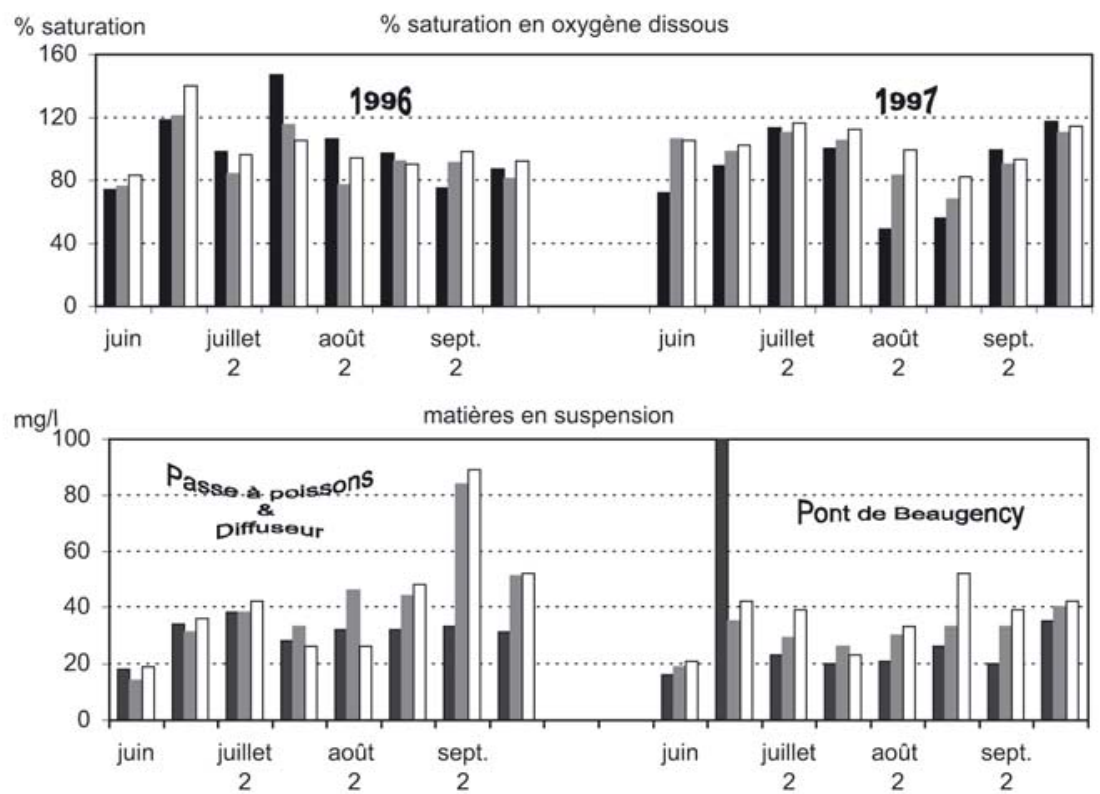

$\mathrm{mg} / \mathrm{l}$

DBO5

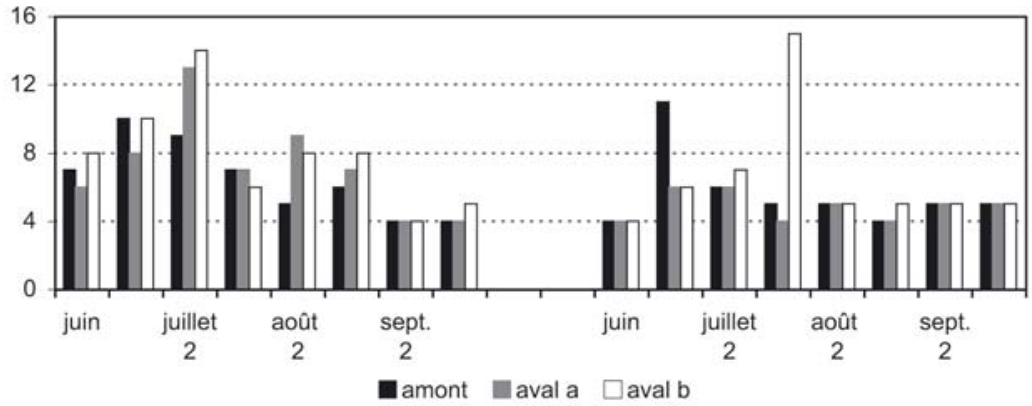

Fig. 16. - Effets des travaux effectués dans le secteur de Saint-Laurent-des-Eaux en 1996-1997, sur la quantité de matières en suspension, sur les teneurs en oxygène dissous et sur la demande biochimique en oxygène.

Fig. 16. - Effect of the earthworks in the riverbed performed in the Saint-Laurent-des-eaux area on the dissolved oxygen and biochemical demand in oxygen (upstream, immediate downstream and distant downstream). 
son de la présence du seuil de la centrale qui a joué un rôle de décanteur. Les algues et les rotifères ont manifestement été très sensibles aux apports de matériel en suspension qui affectent la turbidité, avec ses incidences sur la pénétration de la lumière et l'alimentation des filtreurs; mais les animaux paraissent avoir été davantage perturbés. Ils étaient moins denses (Fig. 11) comparativement à 1995 (les niveaux inférieurs de 2000 et 2001 étant liés à des relations prédateurs-proies).

Les nuisances induites par cet ensemble de travaux ont perduré pendant plusieurs années au niveau des fonds (qui étaient colmatés) et de diverses zones de pollution parfaite- ment visibles dans les secteurs de bordure, dans lesquelles les films bactériens se mêlaient au matériel accumulé (Photo 4). Ce n'est qu'à partir de l'année 2000 que l'atténuation progressive du colmatage des fonds, liée au régime hydrologique, a été visible.

D'un point de vue plus général, au regard des données collectées station par station, les chlorations ont globalement été moins ressenties par les algues que par les rotifères, la baisse de densité des Bacillariophycées planctoniques n'ayant pas affecté très significativement la densité algale. Les rotifères paraissent cependant plus sensibles que les algues; le nombre et la densité des

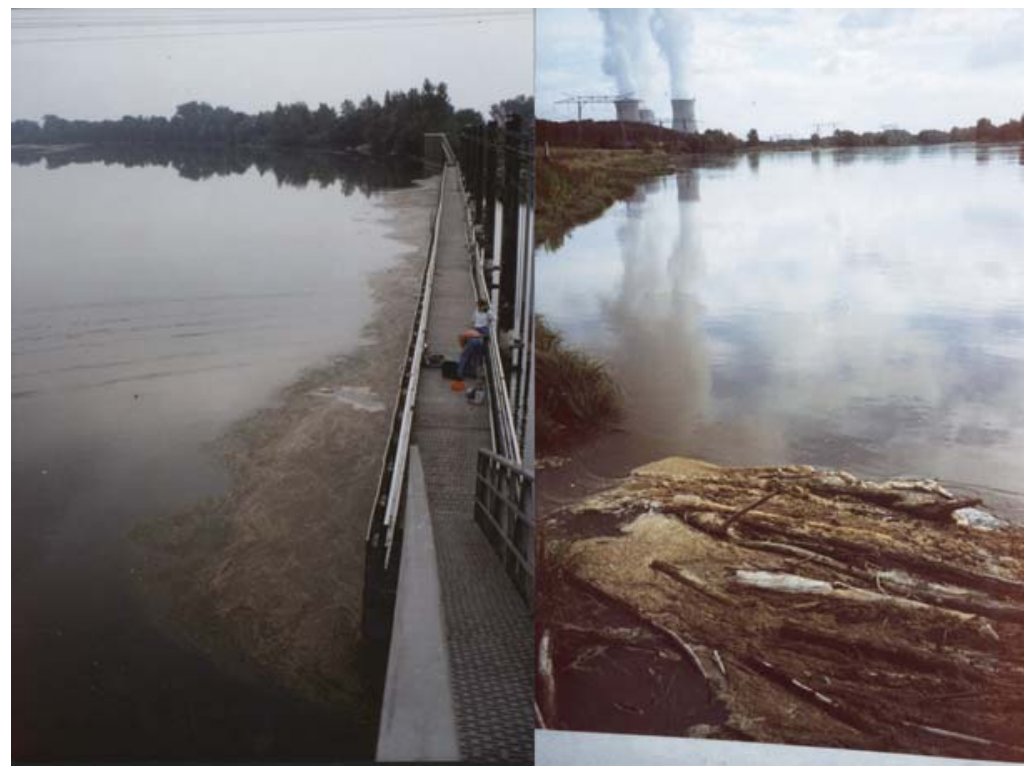

Photo 4. - Salissures et films bactériens. Clichés P. Reyes-Marchant.

Photo 4. - Biological and bacterial films after the excavation works. Snapshot from P. Reyes-Marchant. 
espèces présentes dans le milieu a nettement chuté à Dampierre-enBurly en 1996, année des chlorations, à Saint-Laurent-des-Eaux en 1996 et 1997, au moment des opérations "diffuseur \& passe à poissons ", et ce fut le cas en 1999, au moment des opérations "passe à poissons \& canoë-kayaks " à Dampierre-en-Burly (Fig. 11). Globalement, leur changement de densité ne paraît pas avoir entraîné de répercussions sur la densité algale (l'examen détaillé suggère cependant des incidences sur la distribution de tailles des algues). Cela corrobore leur qualité d'indicateurs (Sladecek, 1983), puisque ce sont eux qui ont été les plus sensibles aux travaux.

\section{CONCLUSION}

Si la nécessité d'évaluer l'impact de l'enrichissement en éléments nutritifs dans les rivières est une préoccupation ancienne (Butcher et al., 1937), la communauté scientifique s'est toujours interrogée sur les facteurs limitant la biomasse phytoplanctonique, alors que les teneurs en $\mathrm{N}$ et $\mathrm{P}$ dans les secteurs aval paraissent suffisantes pour soutenir la production primaire (Dokulil, 1996 ; Rose \& Balbi, 1997).

Les séries temporelles de données sont peu nombreuses dans la littérature et, selon les auteurs, le long terme recouvre des périodes de temps très variables : 10 ans pour le Golfe de Tigullio et illustrant des changements climatiques (Licandro \& Ibanez, 2000) ; 13 ans pour l'Elbe et montrant une amélioration de la qualité de l'eau (Lehmann \& Rode, 2001) ; 20 ans pour la baie de Seine avec un suivi des flagellés, de l'azote et du silicium (Guillaud \& Menesguen, 1998) ou pour la Neuse et soulignant les difficultés à mesurer dans cet intervalle de temps les améliorations apportées par les traitements amont (Stow et al., 2001) ; 24 ans pour faire le point sur les procédés de mesure et d'instrumentation (Garrett, 1999) ; 90 ans en Seine et en Marne, en relation avec le développement socio-économique, et les déficits brutaux en oxygène (Cun \& Vilagines, 1997 ; Cun et al., 1997) ; 100 ans pour des cours d'eau danois, illustrant la perte de richesse de la végétation en liaison avec la modification des habitats (Riis \& Sand-Jensen, 2001). Certains auteurs font également le point après de longues périodes, comme ce fut le cas pour les diatomées de Grand $\mathrm{Ri}$ ver (Rott, 1995). Ceci revient à dire que prévoir le succès d'une restauration nécessite une meilleure connaissance de l'écologie des rivières (Schiemer et al., 1999) et dresser un diagnostic demeure délicat (Barbour et al, 2000).

$\mathrm{Au}$ cours de la synthèse réalisée pour le site de Saint-Laurent-des-Eaux qui s'était arrêtée à l'année 1998, la baisse des teneurs en éléments nutritifs n'était pas encore perceptible de manière significative (Lair \& ReyesMarchant, 2000). C'est dire le caractère relatif de ce qui est nommé « long terme ». Cependant, dans le cas de la Loire Moyenne, il apparaît que vingt 
années ont permis un recul suffisant pour percevoir une évolution de la qualité de l'eau, le changement observé depuis les années 1995-1996 s'étant avéré persistant. II s'est traduit par une baisse des teneurs en oxygène dissous, consécutive à la diminution de la quantité d'algues. Les données du RNB avaient également révélé une chute de la densité algale de $30 \%$ entre 1994 et 1995 à la station de Gien (rapport de synthèse sur le phytoplancton de la Loire, A.E.L.B. 1998) et un processus analogue a été constaté sur la Saône à partir de 1995 également. Cette baisse de la quantité d'algues est associée à la diminution des teneurs en phosphates qui, à certaines dates, sont aux limites de détection des méthodes normalisées d'analyse.

Sur le moyen terme, correspondant aux 7 dernières années d'étude, qui fut caractérisé par des basses eaux, le constat s'avère plus délicat, d'autant que diverses nuisances ont affecté le cours du fleuve et ont inévitablement contribué à une certaine mortalité algale. Cependant, au regard de la série de données correspondant aux années hydrologiquement déficitaires, d'un point de vue fonctionnel, il ressort que les communautés vivantes sont particulièrement résilientes. Enfin, notre attention a été retenue par le rôle des algues dans ce secteur de Loire. En effet, entre fin juin et début octobre, pour la période 1982-2001, les corrélations entre teneurs en oxygène dissous et quantité d'algues étaient positives ( $y$ compris en étiage sévère). Par contre, pour la période 1995-2001, la demande en oxygène s'accentue et l'absence de corrélation entre teneurs en oxygène dissous et quantité d'algues, révèle que les algues ne dominent plus les processus d'oxygénation de l'eau.

La Directive Cadre européenne sur l'Eau fixant pour objectif d'atteindre à l'horizon 2015 le "bon état écologique", cela nécessite d'avoir un état de référence qui, à l'évidence, ne peut être celui de l'origine. Grâce aux programmes de surveillance des centrales nucléaires, nous disposons d'un "premier état de référence », car ayant trait à de nombreux paramètres, celui dressé en 1977-1978 sous conditions extrêmes, et d'un suivi sur vingt ans d'un secteur de fleuve qui intègre l'influence naturelle et anthropique de plus de $500 \mathrm{~km}$ de rivière et d'un bassin versant de l'ordre de $35000 \mathrm{~km}^{2}$. Au regard des résultats obtenus au cours de ces suivis, il est possible de caractériser la qualité de l'eau de la Loire en « période sensible » de la manière suivante :

1) La Loire Moyenne est un milieu naturellement eutrophe, de par sa distance à la source, la nature même de son régime hydraulique et de son lit, et il est difficile de dissocier les apports liés aux diverses activités humaines qui contribuent à son enrichissement, des apports naturels en nutriments. Sur le long terme, en périodes de basses eaux, les algues sont responsables, pour une large part, de l'oxygénation de l'eau. Cependant, les processus physiques conditionnent largement le devenir des communautés et dans l'ensemble, l'abondance des algues est 
aussi contrôlée par les débits. Wehr \& Descy (1998) considèrent que les éléments nutritifs deviennent limitants dans les systèmes qui en reçoivent peu et dont le temps de résidence des eaux est long, soulignant que peu de fleuves combinent ces deux conditions.

Toutefois, la Loire moyenne est un secteur de fleuve peu profond, très productif et en période d'étiage sévère, le développement des algues n'est pas toujours conditionné par les débits (cas de Saint-Laurent-desEaux sur le long terme). Durant les périodes de sècheresse, le lessivage des sols est limité et la charge en phosphore arrivant de l'amont peut devenir limitante. En effet, de 1989 à 1991, une baisse de la quantité d'algues avait été observée, consécutivement à une baisse des flux de phosphates. Mais, avec des débits supérieurs à cette première période, la récente période d'étiages sévères a été associée à une baisse de la quantité d'algues, qui pourrait être attribuable à une limitation par le phosphore.

2) Par voie de conséquence, il s'est produit une baisse des teneurs en oxygène dissous. L'observation visuelle, effectuée régulièrement dans les secteurs prospectés, autorise à dire que le développement des macrophytes immergés, compétiteurs directs des algues, ne paraît pas s'être amplifié. Les dresseines ou les corbicules qui sont des filtreurs puissants n'ont pas encore essaimé, mais selon Khalanski (communication personnelle), il est à craindre que leur in- vasion possible (déjà effective dans la Vienne, un affluent de la Loire) affecte encore plus l'oxygénation des eaux, comme ce fut le cas dans diverses rivières (Bachmann et al., 1995 ; Effler et al., 1996 ; Caraco et al., 1997). S'ajoutant au constat actuel, ce type de phénomène peut avoir des conséquences dramatiques sur la qualité de l'eau.

3) Au point de vue chimique, au regard de la $\mathrm{DBO}_{5}$ et de la DCO dont les valeurs demeurent faibles en été, la charge organique tendrait à diminuer, cependant que la moins bonne efficacité de la production primaire (liée à la baisse de la quantité d'algues) contribuerait à amplifier les processus de décomposition.

4) Si le traditionnel continuum fluvial est globalement manifeste, avec une augmentation de la quantité de matériel vivant entre Dampierre-enBurly et Saint-Laurent-des-Eaux situé $90 \mathrm{~km}$ en aval, la vie des communautés vivantes paraît étroitement liée à la morphologie du lit et de ses marges, spécifique au Val de Loire, d'autant que l'abondant potamoplancton estival est lié à la présence de nombreuses zones d'eaux calmes (Picard, Lair \& Reyes-Marchant, en préparation).

5) Quant à l'état biologique, il faut admettre que si la Loire est un milieu largement eutrophe, "il se porte bien ", les communautés vivantes paraissant bien adaptées à l'enrichissement du milieu. Ce sont en majorité des petits organismes à mode de reproduction binaire ou parthénogénétique et le même schéma de déve- 
loppement se reproduit avec une saisonnalité marquée. Le système biologique est résilient, signe d'un "bon état écologique". Nous venons de voir que les communautés planctoniques végétales résistent très bien aux perturbations de courte durée, elles sont riches en espèces et très diversifiées (et la diversité n'a pas diminué au cours du temps). Il en est de même des communautés planctoniques animales qui, bien que sensibles aux travaux (rotifères Trichocercidae), récupèrent vite et demeurent riches et diversifiées. C'est aussi le cas des diatomées benthiques et des macroinvertébrés benthiques étudiés dans le cadre des suivis écologiques. Autre signe de bonne santé, et bien que la méthode des I.B.G.N. appliquée à ce type de milieu s'avère peu robuste (Lair \& ReyesMarchant, 2000), une note IBGN de 19 , exceptionnelle, liée entre autres à une forte variété taxonomique, a été obtenue en juin 2000.

Au regard des programmes liés à la gestion du bassin versant, une réflexion commune avec les gestionnaires autour de cet ensemble de données, dont quelques traits seulement ont été présentés, permettra d'apporter des éléments de réponse quant au devenir de la qualité des eaux de ce secteur du Val de Loire et au rétablissement d'un « meilleur état écologique ", au sens "lutte contre l'eutrophisation culturelle ». II va sans dire que les nécessaires données ultérieures, recueillies grâce à ces suivis hydroécologiques, contribueront à savoir comment évolue le phénomène de diminution de l'eutrophisation observé depuis 1995-1996.

\section{REFERENCES}

Admiraal W., Mylius S.D., Van Steveninck E. D. \& Tubbing D.M.J., 1993. A model of phytoplankton in the lower River Rhine verified by observed changes in silicate concentration. Journal of Plankton Research, 156 : 659-682.

Bachmann V., Gegielka E., Wagner P., Useglio-Polatera P. \& Moreteau J.C., 1995. Installation de l'Amphipode Corophium curvispinum et de la palourde asiatique Corbicula sp. dans la partie française de la Moselle. Hydroécologie Appl. 7 : 185-191.

Barbour M.T., Swietlik W.F., Jackson S.K., Courtemanch D.L., Davies S.P. \& Yoder C.O., 2000. Measuring the attainment of biological integrity in the USA : A critical element of ecological integrity. Hydrobiologia 422-423 : 453464.

Bunn S.E., \& Davies P.M., 2000. Biological processses in running waters and their implications for the assessment of ecological integrity. Hydrobiologia 422/423 : 61-70.

Butcher R.W, Longwell J. \& Pentelow F.T.K., 1937. Survey of the River Tees. Part III. The Non-Tidal-Reaches Chemical and Biological. Her Majesty's Stationery Office, London, $189 \mathrm{p}$.

Caraco N.F., Cole J.J., Raymond P.A., Strayer D.L., Pace M.L., Findlay S.E.G. \& Fischer D.T., 1997. Zebra mussel invasion in a large, turbid river : phytoplankton response to increased grazing. Ecology 78 : 588-602.

Champ P., 1977. Etude des populations d'un Rotifère épiphyte dans la Loire. Cah. Du Laboratoire d'hydrobiologie de Montereau 4 : 1-64.

Champ P., 1978. Dynamique d'une population d'un Rotifère épiphyte thermo- 
phile (Sinantherina socialis) en présence de pollution thermique. Arch. Hydrobiol. 83 : 213-231.

Comte M.F., 1998. Voyages sur la Loire. Ed. C.L.D., 267 p.

Cun C. \& Vilagines R., 1997. Time series analysis on chlorides, nitrates, ammonium and dissolved oxygen concentrations in the Seine river near Paris. The Sciences of the Total environment 208 : 59-69.

Cun C., Bousquet G. \& Vilagines R., 1997. A 90-year record of water quality data of Paris Seine and Marne rivers. J. Water SRT - Aqua. 46 : 150-164.

Descy J.P., 1992. Eutrophication in the River Meuse. In Sutcliffe, D.W. \& Jones J.G. (Eds). Eutrophication: research and application to water supply. Freshwater Biological Association Ambleside U.K., 130-142.

Dokulil M., 1996. Evaluation of eutrophication potential in rivers. The Danuve example, a review. In B.A. Whitton \& E. Rott (eds). Use of Algae for Monitoring Rivers II. Institut für Botanik, University Innsbruck, Innsbruck, 173-178.

EDF, 1996. Eau et environnement. Guide pratique à l'usage des exploitants des centrales thermiques classiques et nucléaires. Ed. EDF-Production Transport, $143 \mathrm{p}$.

Effler S.W., Brooks C.M., Whitehead K., Wagner B., Doerr S., Perkins M., Siegfried C.A., Walrath L. \& Canale R.P., 1996. Impact of zebra mussel invasion on river water quality. Water Environ. Res. 68 : 205-214.

Essington T.E. \& Carpenter S.R., 2000. Nutrient cycling in lakes and streams : Insights from a comparative analysis. Ecosystems 3/2 : 131-143.

Fénelon P., 1967. Les aménagements de la Loire et de ses affluents. Association pour la Création et le Développement du Centre d'Etudes Supérieures Ligériennes, $1: 21-44$.

Gachon L., 1964. L'eau domestiquée. Lib. Delaunay. Clermont-Ferrand.
Galkovskaya G.A., \& Molotkov D.V. 2001. Species diversity and dominance in the planktonic rotifer community of the Pripyat River in the Chernobyle region (1988-1996). Hydrobiologia 446-447 : 179-185.

Garnier J., Billen G. \& Coste M., 1995. Seasonal succession of diatoms and Chlorophyceae in the drainage network of the Seine River : Observations and modeling. Limnol. Oceanogr. 40 $4: 750-765$.

Garrett T.M. Jr, 1999. Instrumentation, control and automation progress in the united states in the last 24 years. IAWQ, $7 \mathrm{p}$.

J.C., 1980. Physiological processes, nutrient availability and the concept of relative growth rate in marine phytoplankton ecology. In : Primary productivity in the sea. Falkowski P.G. (ed.), Plenum Press, New-York: 179-194.

Gosselain V., Descy J.P. \& Everbecq E., 1994. The phytoplankton community of the river Meuse, Belgium : seasonal dynamics year, 1992, and the possible incidence of zooplankton grazing. Hydrobiologia 289 : 179-191.

Guillaud J.F. \& Menesguen A., 1998. Modelling over twenty years, 1976-1995, of the phytoplanktonic production in the Bay of Seine Fren.Oceanol. Acta $21: 887-906$.

Hildrew A.C., 1996. Whole river ecology. Spatial scale and heterogeneity in the ecology of running waters. Arch. Hydrobiol. Suppl. $113:$ 25-43.

House W.A., Leach D.V. \& Armitage. P.D., 2001. Study of dissolved silicon, and nitrate dynamics in a fresh water stream. Water Res. 35/11: 27492757.

Kelly M.G. \& Whitton B.A., 1998. Biological monotoring of eutrophication in rivers. Hydrobiologia 384 : 55-67.

Köhler J., 1997. Measurement of in situ growth rates of phytoplankton under conditions of simulated turbulence. 
Journal of plankton Research, 19: 262 : 849-862.

Kobayashi T., Shiel R.J., Gibbs P. \& Dixon P.I., 1999. Freshwater zooplankton in the Hawkesbury-Nepean River: Comparison of community structure with other rivers. Hydrobiologia 377 : 133-145.

Lack T.J., 1971. Quantitative studies on the phytoplankton of the rivers thames and kennet at reading. Freshw. Biol., $1: 213-224$.

Lair N., 1975-1976. Projet Alpin O.C.D.E. pour la lutte contre l'eutrophisation. Lacs du Massif Central français. I Protocole de mesures. Ann. Stat. Biol. Besse-en-Chandesse, 10, 92-99.

Lair N., Millerioux E. \& Restituito F., 1978. Examen physicochimique et répartition du plancton de la Loire en période d'étiage (été 1976) au niveau de la centrale nucléaire de St-Laurent-desEaux. Cahiers d'hydrobiologie de Montereau, $6: 53-80$.

Lair N., Pihan J.C. \& Nourrisson M., 1980. Conception et principaux résultats des études hydrobiologiques effectuées sur différents sites électronucléaires. Rev. Gén. Nucléaire, $1: 31-44$.

Lair N. \& Sargos D., 1993. A 10 years study in the middle course of the River Loire. I - Patterns of change in hydrological, physical and chemical variables at the sites of nuclear power plants, in relation to algal biomass. Hydroecol. Appl. 5 : 1-27.

Lair N., Sargos D. \& Reyes-Marchant P., 1996. Synthèse des études hydrobiologiques réalisées en Loire Moyenne au niveau du site nucléaire de Dampierre-en-Burly (France). Hydroécol. Appl. 8 : 35-84.

Lair N. \& Reyes-Marchant P., 1997. The potamoplankton of the Middle Loire : the role of the "moving littoral" in downstream transfer of algae and rotifers. Hydrobiologia 356 : 33-52.

Lair N., Reyes-Marchant P. \& Jacquet V., 1998. Développement du phytoplanc- ton, des Ciliés et des Rotifères sur deux sites de la Loire moyenne (France), en période d'étiage. Annls Limnol. 34 : 35-48.

Lair N., Jacquet V. \& Reyes-Marchant P., 1999. Factors related to autotrophic potamoplankton, heterotrophic protists and micrometazoans abundance at two sites in a lowland temperate river, during low water flow. Hydrobiologia $394: 13-28$.

Lair N. \& Reyes-Marchant P., 2000. Caractéristiques hydroécologiques de la Loire moyenne : bilan des études réalisées de 1977 à 1998 sur le site électronucléaire de Saint-Laurent-des-Eaux (France) et perspectives. Hydroécol. Appl. 12 : 1-65.

Lehmann A. \& Rode M., 2001. Long-term behaviour and cross-correlation water quality analysis of the river Elbe, Germany. Water Res. 35 : 2153-2160.

Levine S.N. \& Schindler D.W., 1999. Influence of nitrogen to phosphorus supply ratios and physicochemical conditions on cyanobacteria and phytoplankton species composition in the Experimental Lakes Area, Canada. Can. J. Fish. Aquatic Sci. 56 : 451-466.

Licandro P. \& Ibanez F., 2000. Changes of zooplankton communities in the Gulf of Tigullio Ligurian Sea, Western Mediterranean, from 1985 to 1995. Influence of hydroclimatic factors. J. Plankton Res. 22/12 : 2225-2253.

Marker A.F.H. \& Collen G.D., 1997. Spatial and temporal characteristics of algae in the River Great Ouse. I. Phytoplankton. Regul. Rivers: Res. Mgmt $13: 219-233$.

O.C.D.E (Organisation de Coopération et Développement Economique), 1982. Eutrophication of Waters. Monitoring, Assessement and Control. Final Report. OCDE Cooperative Programme on Monitoring of Inland Waters. (Eutrophication Control), Environment Directorate, OCDE, Paris. $154 \mathrm{p}$. 
Pearl H.W., 1988. Growth and reproductive strategies of freshwater blue-green algae : cyanobacteria. In Growth and reproductive strategies of freshwater phytoplankton. Ed. Sandgren C.D. Cambridge University press, 261-315.

Pourriot R., Rouyer G. \& Peltier P., 1972. Prolifération de rotifères épiphytes et pollution thermique dans la Loire. Bull. fr. Pisc. 244 : 111-118.

Rahm L., Conley D., Sanden P., Wulff F. \& Stalnacke P., 1996. Time series analysis of nutrient inputs to the Baltic Sea and changing Dsi : DIN ratios. Mar. Ecol. Prog. Ser. 130 : 221-228.

Reynolds C.S. \& Descy J.P., 1996. The production, biomass and structure of phytoplankton in large rivers. Arch. $\mathrm{Hy}$ drobiol. Beih. 113 "Large rivers" 10 : 187-198.

Riis T. \& Sand-Jensen K., 2001. Historical changes in species composition and richness accompanying perturbation and eutrophication of Danish lowland streams over 100 years. Freshw. Biol. 46/2 : 269-280.

Rose M. \& Balbi D., 1997. Rivers Nene and Great Ouse Eutrophication studies: final report. Env. Agency, Petersborough, U.K., 77 p.

Rott E., 1995. Diatoms of the Grand River, Ontario, Canada restudied after 25 Years. Limnologica 25/2 : 165-192.

Schiemer F., Baumgartner C. \& Tockner K., 1999. Restoration of floodplain rivers : the "Danube restoration project". Regul. Rivers: Res. Mgmt. 15 : 231244.

Schulé C.A., 2000. Les situations critiques dans la gestion de la Loire et de son bassin. SEDES - Les Régions françaises face aux extrêmes hydrobiologiques 325 : 232-246.

Servais P., 1989. Bacterioplancton biomass and production in the river Meuse (Belgium). Hydrobiologia 174 : 99-110.

Shannon C.E. \& Weaver W., 1949. The Mathematical Theory of Communication. Univ. Press of Illinois, Urbana 117-127.

Sladecek V., 1983. Rotifers as indicators of water quality. Hydrobiologia 100 : 169-201.

Stow C.A., Borsuk M.E. \& Stanley D.W., 2001. Long-term changes in watershed nutrient inputs and riverine exports in the Neuse River, North Carolina. Water Res. 35 : 1489-1499.

Swan C.M. \& Palmer M.A., 2000. What drives small-scale spatial patterns in lotic meiofauna communities ? Freshw. Biol. 44/1 : 109-121.

Tockner K., Schiemer F., Baumgartner C., Kum G., Weigand E., Zeimuller I. \& Ward J.V., 1999. The Danube restoration project : species diversity patterns across connectivity gradients in the floodplain system. Regul. Rivers: Res. Mgmt 15 : 245-258.

Wehr J.D. \& Descy J.P., 1998. Use of phytoplankton in large river management. J. Phycol. 34 : 741-749.

Weithoff G., Lorke A. \& Walz N., 2000. Effects of water-column mixing on bacteria, phytoplankton and rotifers under different levels of herbivory in a shallow eutrophic lake. Oecologia 125 : 91 100.

Wu J., Buchak E.M., Edinger J.E. \& Kolluru V.S., 2001. Simulation of coolingwater dischanges from power plants. J. Environ. Manage. 61 : 77-92. 
